\author{
Ryerson University \\ Faculty of Engineering and Architectural Science \\ Department of Aerospace Engineering
}

\title{
Controller Tuning and Robustness Testing of Attitude Control Laws \\ for a CubeSat Mission
}

Mohamed Jundi

\author{
AER870 \\ Aerospace Engineering Thesis \\ Faculty Advisor - Dr. Anton de Ruiter
}

April 16, 2021 


\section{Abstract}

The purpose of this project was to create a test environment that can be used to test different controllers and their robustness. In this report, the equations of motion were derived using kinematics, with attitude quaternions, and spacecraft dynamics, with angular velocity and acceleration. The equations were combined and placed into the form of a linearized state-space equation. The different control methods being investigated, Linear Quadratic Regulator (LQR) for the reaction wheel model, and the Bdot with bias controller, were explained and the block diagram for each was shown. To setup the test, the tolerances for the roll, pitch, and yaw, and their rates, were taken from the mission requirement for the ESSENCE mission. The attitude tolerance being $\pm 0.5 \mathrm{deg}$ and the angular rates requirement being $\pm 0.05 \mathrm{deg} / \mathrm{s}$. Then the test setup was further explained. The test is broken up into different scripts and steps:

1. Main run function for simulation. Initializes simulation parameters.

2. Build state-space equation and calculate constant gain matrix.

3. Randomize initial conditions and pass onto simulation.

4. Post-processing and plot generation.

5. Statistics generation.

This robust testing environment was used to test 5 different controllers for the reaction wheel model. Each controller was tested for 200 different simulations, in which the initial attitude, initial angular rates, and the center of mass were randomized. The first controller was successful for 198/200 simulations, where the only failure came from over-saturating the reaction wheels. The next three controllers had a perfect record and were successful for all 200 simulations each. The last controller, had only 71 successful simulations in the set, and a sample of one of the failed simulations was further investigated to see how it failed. 


\section{Acknowledgements}

The author would like to thank Dr.de Ruiter for the opportunity to work on this project, and for his supervision and support throughout the term. Next the author would like to thank Mike Alger and William Travis for their great support and help with the theory and software used in this project. Finally, the author would like to thank Ryerson University and the Aerospace Engineering department for providing the opportunity to work on an Undergraduate Thesis Project. 


\section{Contents}

List of Figures $\quad$ v

List of Tables vii

1 Introduction 2

1.1 Background ........................ 2

2 Equations of Motion $\quad 3$

2.1 Frames of Reference [1] . . . . . . . . . . . . . . . . . . . . . . . . . . .

2.2 Kinematics . . . . . . . . . . . . . . . . . . . . 4

2.3 Dynamics . . . . . . . . . . . . . . . . 6

2.4 Linear Set of Equations . . . . . . . . . . . . . . . 8

3 Control Methodology 10

3.1 Linear Quadratic Regulator (LQR) For Reaction Wheels . . . . . . 10

3.2 Augmentation of Integral Control Gain . . . . . . . . . . . . . . . . 12

3.3 B-dot with Bias Gain Tuning . . . . . . . . . . . . . . . 12

4 Software Setup 14

4.1 Existing Model . . . . . . . . . . . . . . . . . . . 14

4.2 Test $\operatorname{Setup} \ldots \ldots \ldots \ldots \ldots$

4.3 Results ............................ 19

4.3.1 Simulation Set $1 \ldots \ldots . \ldots . \ldots 20$

4.3 .2 Simulation Set $2 \ldots \ldots \ldots . \ldots . \ldots . \ldots 25$

4.3 .3 Simulation Set $3 \ldots \ldots \ldots$

4.3 .4 Simulation Set $4 \ldots \ldots \ldots \ldots$

4.3 .5 Simulation Set $5 \ldots \ldots . \ldots . \ldots 40$

5 Conclusion $\quad 46$

$\begin{array}{lll}6 & \text { Bibliography } & 47\end{array}$ 
A Linearization of A Matrix 


\section{List of Figures}

2.1.1 BOF Reference Frame [1] . . . . . . . . . . . . . . 4

3.3.1 Bdot With Bias Controller Block Diagram . . . . . . . . . . . . 13

4.1.1 Full Reaction Wheel Test Model . . . . . . . . . . . . . . . . . . . 15

4.1 .2 Test Harness . . . . . . . . . . . . . . . . . . . . . . . . 16

4.1 .3 Controller Block . . . . . . . . . . . . . . . . . 16

4.2 .1 Software Flowchart . . . . . . . . . . . . . . . . . . . . . 19

4.3.1 Initial Errors for Each Simulation - Set 1 . . . . . . . . . . . 21

4.3.2 Average Errors for Each Simulation - Set 1 . . . . . . . . . . . . 21

4.3.3 Maximum Control Inputs - Set 1 . . . . . . . . . . . . . . . 22

4.3.4 Maximum and Minimum Reaction Wheel Speed - Set 1 . . . . . . 22

4.3.5 Maximum and Minimum Reaction Wheel Speed [RPM]- Set 1 . . 23

4.3.6 Center of Mass Distribution - Set 1 . . . . . . . . . . . . . 23

4.3.7 Summary of Results - Set 1 . . . . . . . . . . . . . . 24

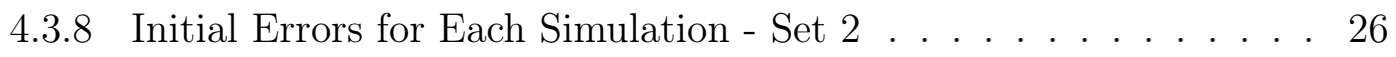

4.3.9 Average Errors for Each Simulation - Set 2 . . . . . . . . . . 26

4.3.10 Maximum Control Inputs - Set 2 . . . . . . . . . . . . . . . . . . 27

4.3.11 Maximum and Minimum Reaction Wheel Speed - Set 2 . . . . . 27

4.3.12 Maximum and Minimum Reaction Wheel Speed [RPM]- Set 2 . 28

4.3.13 Center of Mass Distribution - Set $2 \ldots$. . . . . . . . . . . 28

4.3.14 Summary of Results - Set 2 . . . . . . . . . . . . . . . . . . . . . 29

4.3.15 Initial Errors for Each Simulation - Set 3 . . . . . . . . . . . . 31

4.3.16 Average Errors for Each Simulation - Set 3 . . . . . . . . . . . . 31

4.3.17 Maximum Control Inputs - Set 3 . . . . . . . . . . . . . . . . . 32

4.3.18 Maximum and Minimum Reaction Wheel Speed - Set 3 . . . . . . 32

4.3.19 Maximum and Minimum Reaction Wheel Speed [RPM]- Set 3 . . 33

4.3.20 Center of Mass Distribution - Set 3 . . . . . . . . . . . . . . 33

4.3.21 Summary of Results - Set 3 . . . . . . . . . . . . . . 34

4.3.22 Initial Errors for Each Simulation - Set 4 . . . . . . . . . . . . 36 
4.3.23 Average Errors for Each Simulation - Set 4 . . . . . . . . . . . 36

4.3.24 Maximum Control Inputs - Set 4 . . . . . . . . . . . . . . . 37

4.3.25 Maximum and Minimum Reaction Wheel Speed - Set 4 . . . . . . 37

4.3.26 Maximum and Minimum Reaction Wheel Speed [RPM]- Set 4 . . 38

4.3.27 Center of Mass Distribution - Set 4 . . . . . . . . . . . 38

4.3.28 Summary of Results - Set 4 . . . . . . . . . . . . . . . . . 39

4.3.29 Initial Errors for Each Simulation - Set 5 . . . . . . . . . . . . . 41

4.3.30 Average Errors for Each Simulation - Set 5 . . . . . . . . . . . . . 41

4.3 .31 Maximum Control Inputs - Set 5 . . . . . . . . . . . . . . . . . 42

4.3.32 Maximum and Minimum Reaction Wheel Speed - Set 5 . . . . . . 42

4.3.33 Maximum and Minimum Reaction Wheel Speed [RPM]- Set 5 . . 43

4.3.34 Center of Mass Distribution - Set 5 . . . . . . . . . . . . . 43

4.3.35 Summary of Results - Set 5 . . . . . . . . . . . . . . . . . 44

4.3.36 Attitude and Rates Throughout Orbit for Simulation 5 in Set 5 . 45 


\section{List of Tables}

4.1 RPY and Rates Tolerances . . . . . . . . . . . . . . . 17

4.2 Summary of Results for Simulation Set 1 . . . . . . . . . . . . 20

4.3 Summary of Results for Simulation Set 2 . . . . . . . . . . . 25

4.4 Summary of Results for Simulation Set 3 . . . . . . . . . . . . . 30

4.5 Summary of Results for Simulation Set 4 . . . . . . . . . . . . . 35

4.6 Summary of Results for Simulation Set 5 . . . . . . . . . . . . . . . 40

4.7 Simulation 5 Initial Conditions . . . . . . . . . . . . . . . . 44 


\section{Nomenclature}

\section{Acronyms}

ADCS Attitude Determination and Control System

BOF Spacecraft Body Frame

CoM Center of Mass

$C S A$ Canadian Space Agency

ECEF Earth Centered Earth Fixed Frame

ECI Earth Centered Inertial Frame

ESSENCE Educational Space Science and ENgineering CubeSat Mission

ISS International Space Station

$L Q R$ Linear-Quadratic Regulator

$O R B$ Orbital Frame

RPY Roll, Pitch, Yaw

$S C \quad$ Spacecraft

SRP Solar Radiation Pressure

TER Terrestrial Frame

\section{Matrices and Vectors}

$\boldsymbol{A} \quad$ Upper case, bold font expressed matrix

$\boldsymbol{I}_{\mathbf{3} \times \mathbf{3}}$ Three by three identity matrix

$\boldsymbol{v} \quad$ Lower case, bold font expresses vector

$\dot{v} \quad$ Lower case, with dot above expresses time rate of change

$\hat{\boldsymbol{u}} \quad$ Unit vector

$\boldsymbol{v}^{\times} \quad$ Cross product matrix representation of vector 


\section{List of Symbols}

$\dot{\boldsymbol{\omega}}_{\boldsymbol{B I}}^{B} \quad$ Angular acceleration of body frame relative to inertial frame, expressed in the body frame

$\dot{\boldsymbol{x}} \quad$ Rate of change of state vector

$\boldsymbol{\omega}_{B \boldsymbol{I}}^{B} \quad$ Angular velocity of body frame relative to inertial frame, expressed in the body frame

$\boldsymbol{\tau}_{\text {dis }}$ Disturbance torque in body frame

$\tau_{\boldsymbol{g g}} \quad$ Gravity gradient torque in body frame

$\boldsymbol{\tau}_{\boldsymbol{m a g}}$ magnetic dipole torque in body frame

$\boldsymbol{\tau}_{\boldsymbol{r} \boldsymbol{w} \boldsymbol{l}}$ Reaction wheel torque in body frame

$\boldsymbol{\tau}_{\text {tot }}$ Total torque acting on spacecraft

$\boldsymbol{A} \quad$ Linear A matrix

$\boldsymbol{B} \quad$ Linear B matrix

b Magnetic field vector

$\boldsymbol{J} \quad$ Spacecraft inertia matrix

$\boldsymbol{K} \quad$ Static gain matrix for optimal control from LQR

$\boldsymbol{K}_{\text {dot }}$ Constant gain matrix for Bdot controller

$\boldsymbol{m}_{\boldsymbol{c m d}}$ Command torque vector

$\boldsymbol{P}$ Positive-definite Hermitian or real symmetric matrix

$\boldsymbol{Q}$ Positive-definite Hermitian or real symmetric matrix. Weight Matrix on the states

$\boldsymbol{q}$ Attitude quaternion

$\boldsymbol{r}_{\boldsymbol{B}} \quad$ Position vector of spacecraft

$\boldsymbol{R}$ Positive-definite Hermitian or real symmetric matrix. Weight Matrix on the control input

$\boldsymbol{u}(\boldsymbol{t})$ Control input

$\epsilon \quad$ Vector component of quaternion 
$\eta \quad$ Scalar Component of quaternion

$\mu \quad$ Gravitational Constant

$\phi \quad$ Angle of rotation through unit quaternion rotation

$i, j, k$ Hyper imaginary numbers

$J \quad$ Cost function 


\section{Chapter 1}

\section{Introduction}

\subsection{Background}

This project is part of the research and development in the Educational Space Science and Engineering CubeSat Mission (ESSENCE), by the Canadian Space Agency (CSA). The ESSENCE mission consists of a $2 \mathrm{U}$-CubeSat that will be launched by the International Space Station (ISS). Some of the main objectives of the mission are to demonstrate different attitude control methods, and to monitor the thawing of permafrost and ice in the arctic region [2]. This project is specifically part of work done by the Attitude Determination and Control System (ADCS) team.

The primary goal of this project is to test different under-actuated control laws. To support the development of the test, several standard control laws are used. The first part of the project is dealing with and LQR controller with reaction wheels set in a 3-wheel orthogonal configuration. The report will show the development of a Monte Carlo test script to test a set of control gains over a wide range of different random conditions. Parameters such as initial attitude states, angular rates, and mass distribution will be randomized. The simulation passes the results through a pass-fail checker and plots useful parameters to monitor any anomalies. This test is to tune and verify controllers across different initial conditions and variations in mass and inertia. The second control law investigated in this report is the Bdot with bias. This section builds on existing Bdot control methods done by the team, and introduces a method to test for adding a bias dipole moment to the model, which can help coarsely point align the spacecraft with respect to the magnetic field to help in early commissioning of the spacecraft. 


\section{Chapter 2}

\section{Equations of Motion}

This chapter deals with the equations of motion relative to the spacecraft in Low Earth Orbit (LEO). It will begin with setting the frames of references used. Next the kinematics and the attitude quaternions are discussed and further explained. Then the dynamics relating the torques and the angular velocity and acceleration are discussed. The equations are then combined to show the non-linear state-space equation for the system. Finally, the linearized equations for the 3 reaction wheels orthogonal configuration are presented.

\subsection{Frames of Reference [1]}

It is important to state the frames of references used in this report, which are directly taken from the requirements document for the ESSENCE project, and are stated as follows:

\section{- Earth Centered Inertial Frame (ECI)}

Origin at mass center of Earth

$X$-axis points towards the vernal equinox at J2000 epoch

$Z$-axis points towards polar axis

$Y$-axis completes the right handed coordinate frame

\section{- Orbital Frame (ORB)}

Origin at spacecraft center of mass (CoM)

$X$-axis is the orbit radial unit vector (EARTH CoM to SC CoM)

$Z$-axis is the orbit normal unit vector

$Y$-axis completes the right-handed coordinate frame 


\section{- Terrestrial Frame (TER)}

Origin at mass center of Earth

$X$-axis through $0^{\circ}$ latitude, $0^{\circ}$ longitude

$Z$-axis points to the geographic north pole

$Y$-axis completes the right-handed coordinate frame

\section{- Spacecraft Body Frame (BOF)}

Origin at spacecraft center of mass (CoM)

$X$-axis is the longitudinal axis

$Z$-axis TBD based on mechanical drawings

$Y$-axis TBD based on mechanical drawings

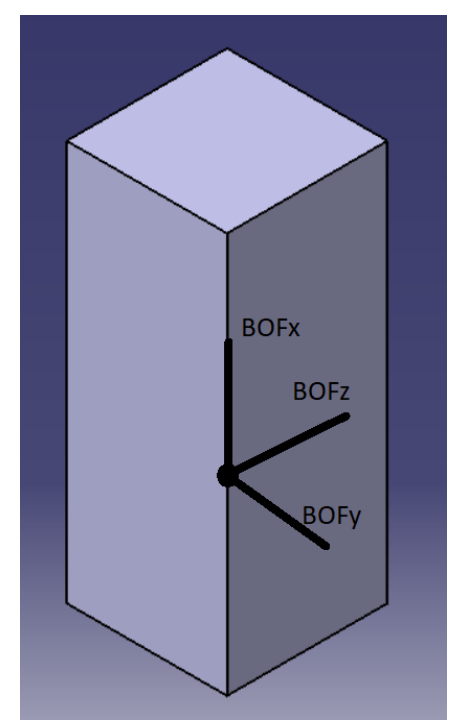

Figure 2.1.1: BOF Reference Frame [1]

\section{$2.2 \quad$ Kinematics}

Before getting into the attitude control of a spacecraft, it is crucial to model the spacecraft kinematics and derive the equations of motion from there. The spacecraft kinematics in this report are modelled and represented using quaternions, in order to avoid running into angle singularity issues. Quaternions are 4 dimensional vectors, consisting of a scalar component and a vector component. In this report, the quaternion is represented using a scalar first representation. This is primarily done in order to keep consistant with the other reports in this project. The spacecraft kinematics can be represented in the following equations: 


$$
\mathbf{q}=\left[\begin{array}{l}
\eta \\
\boldsymbol{\epsilon}
\end{array}\right]=\left[\begin{array}{c}
\eta \\
\epsilon_{x} \\
\epsilon_{y} \\
\epsilon_{z}
\end{array}\right]=\eta+i \epsilon_{x}+j \epsilon_{y}+k \epsilon_{z}
$$

Where $i, j$, and $k$ are imaginary numbers and satisfy the following relations and conditions:

$$
\begin{array}{r}
i^{2}=j^{2}=k^{2}=-1 \\
i j=-j i=k \\
j k=-k j=i \\
k i=-i=j
\end{array}
$$

Where $\eta$ is the scalar component and $\epsilon_{x}, \epsilon_{y}$, and $\epsilon_{z}$ are the vector components and can be calculated and expressed in the following manner:

$$
\begin{array}{r}
\eta=\cos \frac{\phi}{2} \\
\epsilon_{x}=u_{x} \sin \frac{\phi}{2} \\
\epsilon_{y}=u_{y} \sin \frac{\phi}{2} \\
\epsilon_{z}=u_{z} \sin \frac{\phi}{2}
\end{array}
$$

Equation (2.2.2) shows that the unit quaternion can be obtained using a unit vector $\mathbf{u}=\left[\begin{array}{l}u_{x} \\ u_{y} \\ u_{z}\end{array}\right]$ and a rotation angle $\phi$.

In order to start building the state space vector, the time derivatives of both the scalar and the vector components of the quaternion can be expressed in the following non-linear equations [3]:

$$
\dot{\eta}=-\frac{1}{2} \boldsymbol{\epsilon}^{\boldsymbol{T}} \boldsymbol{\omega}_{\boldsymbol{B I}}^{B}
$$




$$
\dot{\boldsymbol{\epsilon}}=\frac{1}{2}\left(\boldsymbol{\epsilon}^{\times}+\eta \boldsymbol{I}_{\mathbf{3} \times \mathbf{3}}\right) \boldsymbol{\omega}_{\boldsymbol{B} \boldsymbol{I}}^{B}
$$

Where $\boldsymbol{I}_{\mathbf{3} \times \mathbf{3}}$ is a $3 \times 3$ identity matrix, and $\boldsymbol{\omega}_{\boldsymbol{B} \boldsymbol{I}}^{B}$ is the angular rate of the body frame with respect to the inertial frame, represented in the body frame. Thus giving the final non-linear form of the kinematics as follows:

$$
\dot{\mathbf{q}}=\left[\begin{array}{c}
\dot{\eta} \\
\dot{\epsilon}
\end{array}\right]=\left[\begin{array}{c}
-\frac{1}{2} \boldsymbol{\epsilon}^{T} \boldsymbol{\omega}_{B \boldsymbol{I}}^{B} \\
\frac{1}{2}\left(\epsilon^{\times}+\eta \boldsymbol{I}_{3 \times 3}\right) \boldsymbol{\omega}_{B I}^{B}
\end{array}\right]
$$

\subsection{Dynamics}

In order to complete the state vector, the angular rate of the spacecraft is also required. This can be obtained by modelling the rotational dynamics of the spacecraft. In order to derive the equation for the angular rate of the spacecraft, the total external torque acting on the spacecraft is taken into consideration. This can be expressed in equation 2.3.1 [4]:

$$
\tau_{t o t}=J \dot{\omega}_{B I}^{B}+\omega_{B I}^{B} \times J \omega_{B I}^{B}
$$

Where $\boldsymbol{J}$ is the spacecraft inertia matrix, $\boldsymbol{\omega}_{B \boldsymbol{I}}^{B}$ is the angular acceleration of the BOF relative to the ECI, and $\boldsymbol{\omega}_{B \boldsymbol{I}}^{B}$ is the angular velocity of the BOF relative to the ECI, both expressed in the BOF.

The total torque acting on the spacecraft can be expressed in the individual torques that are a resultant of the natural forces acting on the spacecraft. These can be listed as the following:

- Torque due to the gravity gradient acting on the spacecraft

- Torque due to the interaction between the Earth magnetic field and the magnetic dipoles on board the spacecraft.

- The disturbance torques due to the aerodynamic drag and the SRP acting on the spacecraft

- The torque applied to the spacecraft by the reaction wheels 
The total torque can be expressed in the following equation:

$$
\tau_{t o t}=\tau_{g g}+\tau_{m a g}+\tau_{d i s}+\tau_{r w l}
$$

Where the gravity gradient torque and the magnetic torque can be expressed in equations (2.3.3) and (2.3.4) respectively

$$
\begin{gathered}
\boldsymbol{\tau}_{\boldsymbol{g} \boldsymbol{g}}=\frac{3 \mu}{\left\|\boldsymbol{r}_{B}(t)\right\|^{3}} \boldsymbol{r}_{B} \times \boldsymbol{J} \boldsymbol{r}_{\boldsymbol{B}} \\
\boldsymbol{\tau}_{\boldsymbol{m a g}}=\boldsymbol{m}_{\boldsymbol{B}} \times \boldsymbol{b}_{\boldsymbol{B}}
\end{gathered}
$$

To get an equation for the angular acceleration of the BOF, relative to the ECI, expressed in the BOF, can be obtained by substituting equation 2.3.2 into equation 2.3.1 and rearranging for $\boldsymbol{\omega}_{B \boldsymbol{I}}^{B}$ :

$$
\dot{\omega}_{B I}^{B}=J^{-1}\left(-\omega_{B I}^{B} \times J \omega_{B I}^{B}+\tau_{g g}+\tau_{m a g}+\tau_{d i s}+\tau_{r w l}\right)
$$

Now equations (2.2.6) and (2.3.5) can be combined and rearranged and put into a state-space equation of the form:

$$
\dot{\boldsymbol{x}}=\boldsymbol{A x}+\boldsymbol{B u}
$$

Where the $\mathrm{x}$ vector representing the states that are to be controlled and it's derivative are the following:

$$
\begin{aligned}
& x=\left[\begin{array}{c}
q \\
\omega
\end{array}\right]=\left[\begin{array}{c}
\eta \\
\epsilon \\
\omega
\end{array}\right] \\
& \dot{x}=\left[\begin{array}{c}
\dot{q} \\
\dot{\omega}
\end{array}\right]=\left[\begin{array}{c}
\dot{\eta} \\
\dot{\boldsymbol{\epsilon}} \\
\dot{\omega}
\end{array}\right]
\end{aligned}
$$

and the control input is a torque vector: 


$$
\boldsymbol{u}=\left[\begin{array}{c}
\tau_{x} \\
\tau_{y} \\
\tau_{z}
\end{array}\right]
$$

The non linear A and B matrix are expressed in equations (2.3.9) and (2.3.10)

$$
\begin{aligned}
& A=\left[\begin{array}{c}
-\frac{1}{2} \boldsymbol{\epsilon}^{\boldsymbol{T}} \boldsymbol{\omega}_{B I}^{B} \\
\frac{1}{2}\left(\epsilon^{\times}+\eta \boldsymbol{I}_{3 \times \mathbf{3}}\right) \boldsymbol{\omega}_{B I}^{B} \\
\boldsymbol{J}^{-1}\left(-\boldsymbol{\omega}_{B \boldsymbol{I}}^{B} \times \boldsymbol{J} \boldsymbol{\omega}_{B \boldsymbol{I}}^{B}+\boldsymbol{\tau}_{g g}+\boldsymbol{\tau}_{d i s}\right)
\end{array}\right] \\
& B=\left[\begin{array}{c}
0_{1 \times 3} \\
0_{3 \times 3} \\
\boldsymbol{J}^{-1}
\end{array}\right]
\end{aligned}
$$

\subsection{Linear Set of Equations}

The linearization of the A matrix is taken from reference [5] and the linearized B matrix is taken from reference [1]. The linearization of the A matrix is included in Appendix A:

$$
\begin{gathered}
A=\left[\begin{array}{cccccc}
0 & \omega_{0} & 0 & \frac{1}{2} & 0 & 0 \\
-\omega_{0} & 0 & 0 & 0 & \frac{1}{2} & 0 \\
0 & 0 & 0 & 0 & 0 & \frac{1}{2} \\
0 & 0 & 0 & 0 & \sigma_{x} \omega_{0} & 0 \\
0 & -6 \omega_{0}^{2} \sigma_{y} & 0 & \sigma_{y} \omega_{0} & 0 & 0 \\
0 & 0 & 6 \omega_{0}^{2} \sigma_{y} & 0 & 0 & 0
\end{array}\right] \\
B=\left[\begin{array}{ccc}
0 & 0 & 0 \\
0 & 0 & 0 \\
0 & 0 & 0 \\
\frac{1}{J_{x x}} & 0 & 0 \\
0 & \frac{1}{J_{y y}} & 0 \\
0 & 0 & \frac{1}{J_{z z}}
\end{array}\right]
\end{gathered}
$$

Where $\omega_{0}$ and $\sigma$ are defined in equations (2.4.3) and (2.4.4): 


$$
\begin{gathered}
\omega_{0}=\sqrt{\frac{\mu}{r^{3}}} \\
\sigma=\left[\begin{array}{c}
\sigma_{x} \\
\sigma_{y} \\
\sigma_{z}
\end{array}\right]=\left[\begin{array}{l}
\frac{I_{y y}-I_{z z}}{I_{x x}} \\
\frac{I_{z z}-I_{x x}}{I_{y y}} \\
\frac{I_{x x}-I_{y y}}{I_{z z}}
\end{array}\right]
\end{gathered}
$$




\section{Chapter 3}

\section{Control Methodology}

This chapter goes over the control methods used in this project. First is the static gain controller used for the reaction wheel model, which is derived using a Linear Quadratic Regulator (LQR) tuning method. The second one is the B-dot with bias controller, which uses a constant gain matrix, magnetic field, and a bias to dampen the spacecraft rates.

\subsection{Linear Quadratic Regulator (LQR) For Reac- tion Wheels}

The main control law used in this project is the Linear Quadratic Regulator control law. This methodology is used to obtain the appropriate static gain matrix. To begin, the linearized state-space equation in the form of equation (2.3.6) is considered:

$$
\dot{x}=A x+B u
$$

Where the feedback gain matrix $\mathrm{K}$ and feedback control action can be expressed in the following form:

$$
u(t)=-K x(t)
$$

Where in the case of the reaction wheel model, the control/ input vector consists of the wheel torques: 


$$
\boldsymbol{u}=\left[\begin{array}{c}
\tau_{r w l, x} \\
\tau_{r w l, y} \\
\tau_{r w l, z}
\end{array}\right]
$$

To solve the optimal control problem, the controller chooses a $\boldsymbol{u}(\boldsymbol{t})$ to minimize the following cost function: [6]

$$
J=\int_{0}^{\infty}\left(x^{T} Q x+u^{T} R u\right) d t
$$

Where the $\boldsymbol{Q}$ and $\boldsymbol{R}$ are positive definite matrices that the user prescribes. Before solving for the gain matrix $\boldsymbol{K}$, the Matrix Algebraic Riccati Equation (MARE) is to be solved for a positive matrix $\boldsymbol{P}$, shown in equation (3.1.4):

$$
A^{T} P+P A-P B R^{-1} B^{T} P+Q=0
$$

Once the $\boldsymbol{P}$ matrix is solved from equation (3.1.4), the feedback law gives the following equation,

$$
u(t)=-R^{-1} B^{T} P x(t)
$$

And substituting equation (3.1.1) into (3.1.5), the optimal gain matrix $\boldsymbol{K}$ can be determined to be,

$$
K=R^{-1} B^{T} P
$$

Although there are numerical methods to go through the procedure outlined above, in solving for $\boldsymbol{P}$ and then calculating the gain matrix, it is of common practice to use standard methods directly using tools and software such as MATLAB, with the function:

$$
\boldsymbol{K}=\operatorname{lqr}(\boldsymbol{A}, \boldsymbol{B}, \boldsymbol{Q}, \boldsymbol{R})
$$




\subsection{Augmentation of Integral Control Gain}

In order to reduce the steady state error of the attitude signal, the state vector was further manipulated and augmented to include an integral of the attitude error, shown in equation (3.2.1) [7]

$$
\overline{\boldsymbol{x}}=\left[\begin{array}{c}
\int_{t}^{t+1} \boldsymbol{\epsilon}_{B N} \\
\boldsymbol{\epsilon}_{B N} \\
\boldsymbol{\omega}_{B N}^{B}
\end{array}\right]
$$

Although the augmentation shown in equation (3.2.1) does not change the derived $\mathrm{A}$ and $\mathrm{B}$ matrices from equations (2.4.1) and (2.4.2), the augmentation must be taken into consideration in the new altered state space representation. This is shown in equations (3.2.2) and (3.2.3) below. [7]

$$
\begin{gathered}
\overline{\boldsymbol{A}}=\left[\begin{array}{ccc}
\mathbf{0}_{3 \times 3} & \boldsymbol{I}_{3 \times 3} & \mathbf{0}_{3 \times 3} \\
\mathbf{0}_{3 \times 3} & \mathbf{0}_{3 \times 3} & \boldsymbol{A}
\end{array}\right] \\
\overline{\boldsymbol{B}}=\left[\begin{array}{c}
\boldsymbol{0}_{3 \times 3} \\
\boldsymbol{B}
\end{array}\right]
\end{gathered}
$$

This augmentation changes the previously stated state space equation, as it adds an extra integral gain to the system while calculating the $\boldsymbol{K}$ gain matrix from in the LQR method stated above. The new altered state space representation of the system can be expressed in equation (3.2.4) below.

$$
\dot{\overline{\boldsymbol{x}}}=\overline{\boldsymbol{A}} \overline{\boldsymbol{x}}-\overline{\boldsymbol{B}} \boldsymbol{K} \overline{\boldsymbol{x}}
$$

\subsection{B-dot with Bias Gain Tuning}

The second part of the project is to look at the B-dot gain tuning. This method requires the measurement of the magnetic field rate $\dot{\boldsymbol{b}}$, taken by the magnetometer, and multiplies it by a constant gain matrix $\boldsymbol{K}_{d o t}$. This gives the dipole moment of the magnetic controller. In order to further modify this model, a bias vector is added, and so the final equation is represented in equation (3.3.1) [3] 


$$
\boldsymbol{m}_{c m d}=-\boldsymbol{K}_{d o t} \dot{\boldsymbol{b}}+\boldsymbol{m}_{\text {bias }}
$$

and the constant gain matrix takes of the form shown in equation (3.3.2)

$$
\boldsymbol{K}_{d o t}=\left[\begin{array}{ccc}
k_{d o t, x} & 0 & 0 \\
0 & k_{d o t, y} & 0 \\
0 & 0 & k_{d o t, z}
\end{array}\right]
$$

The block diagram for the b-dot with bias controller is fairly similar to the original bdot controller block diagram. The rate of the magnetic field rate in the body frame is and multiplied by the gain matrix, and then the bias is added. The block diagram is shown in figure (3.3.1).

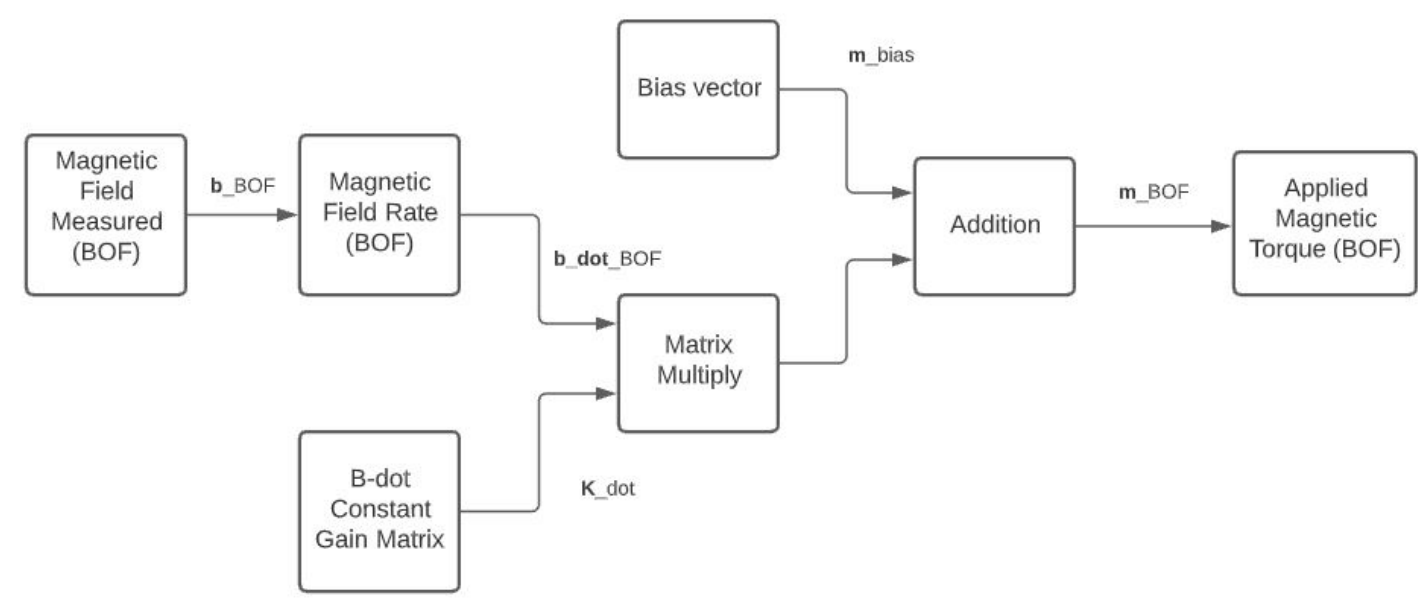

Figure 3.3.1: Bdot With Bias Controller Block Diagram 


\section{Chapter 4}

\section{Software Setup}

This chapter deals with the SIMULINK and MATLAB model setup. The existing SIMULINK models are shown, which further help visualize the test harness and the controller setup. Next the simulation test software is further discussed, as it is broken down into different scripts, each having a certain functionality in the test. Finally, the results for the tests conducted are displayed.

\subsection{Existing Model}

A SIMULINK model covering the dynamics and control of ESSENCE was provided. The full test model from highest level up can be seen in figure (4.1.1) 


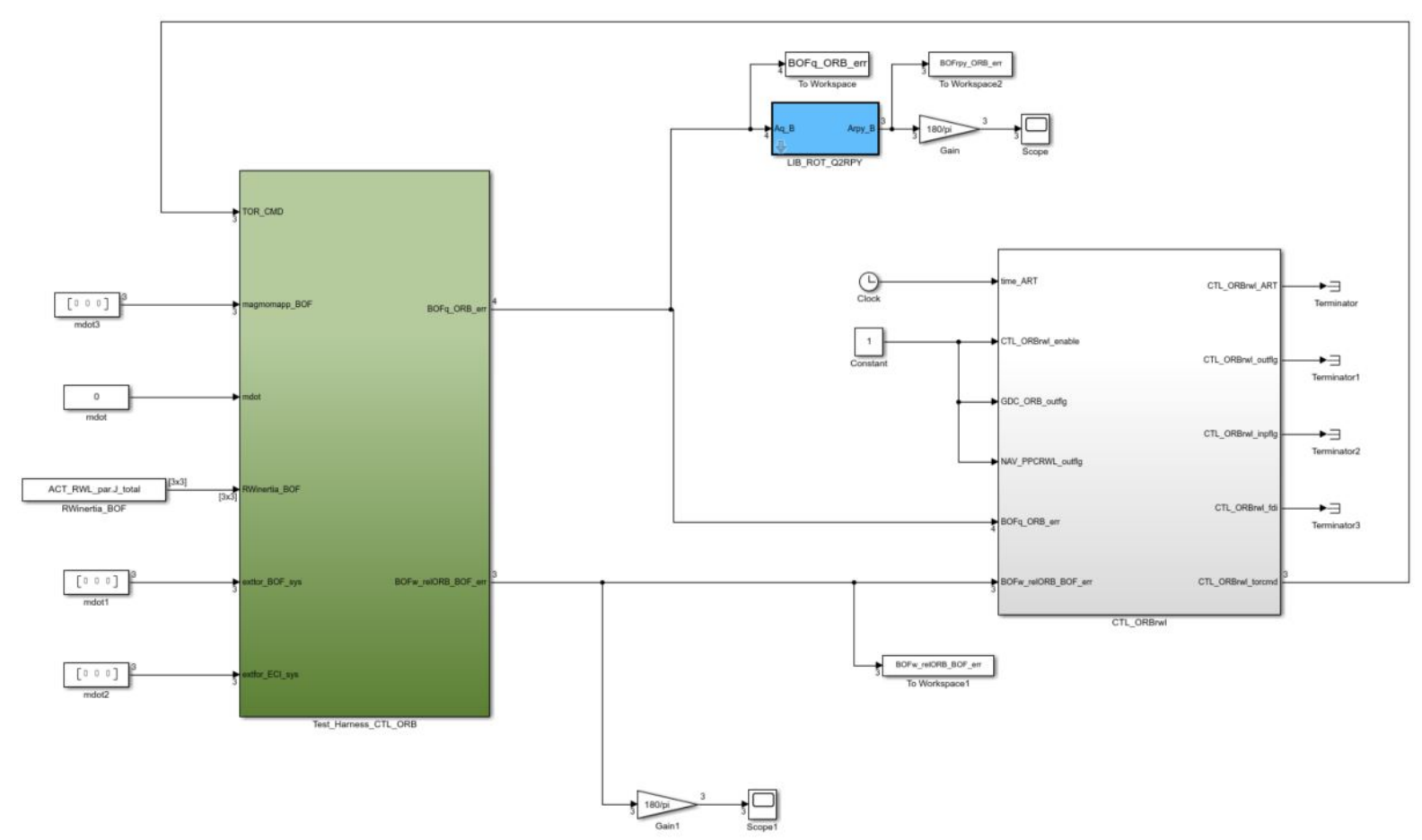

Figure 4.1.1: Full Reaction Wheel Test Model

Where the green block is the test harness and the white block is the unit test.

The test harness is then broken down into the model seen in figure (4.1.2). With the furthest block to the left models the dynamics of the reaction wheels, and so the reaction wheel speed can be monitored in order to ensure the max speed is not exceeded. The middle blue block has all the dynamics of the satellite. All the outputs from that block are passed onto a separate block where they are passed onto the workspace for use. The far right white block is a function to find the error required for analysis. 


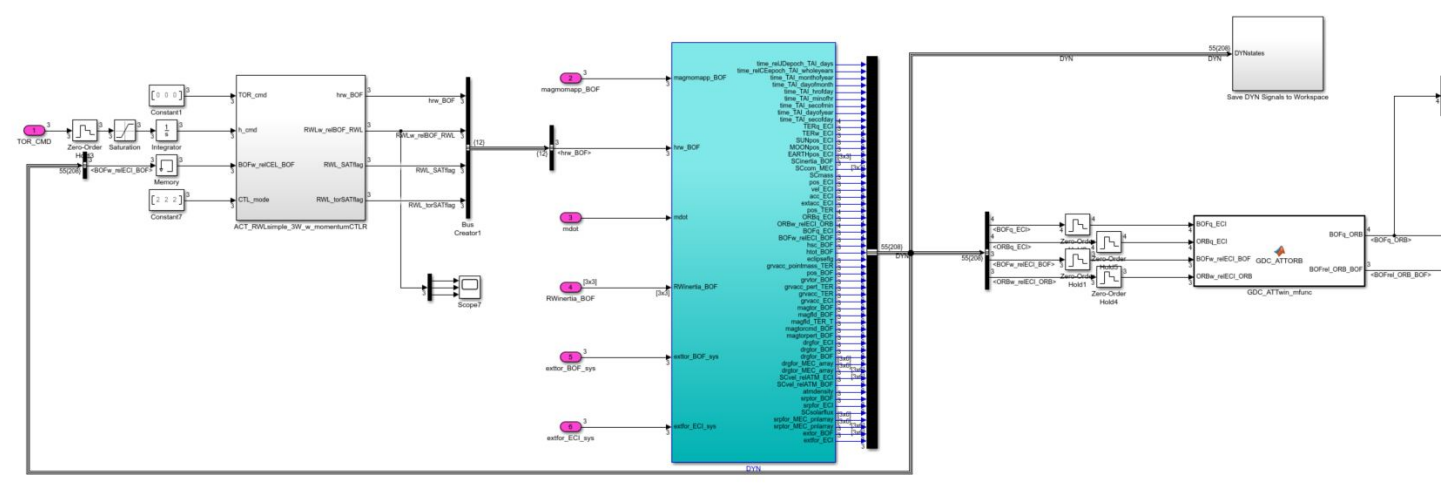

Figure 4.1.2: Test Harness

The controller block is shown in figure (4.1.3). This shows how the quaternion and the angular rate errors are taken as inputs and multiplied by the constant gain matrix, $\boldsymbol{K}$, which is obtained using the LQR control method outlined earlier in this report. The discrete time integrator shown in the figure also represents the augmentation explained in the sections above. The terminated signal is the scalar component of the attitude quaternion, which gets neglected in the linearization process.

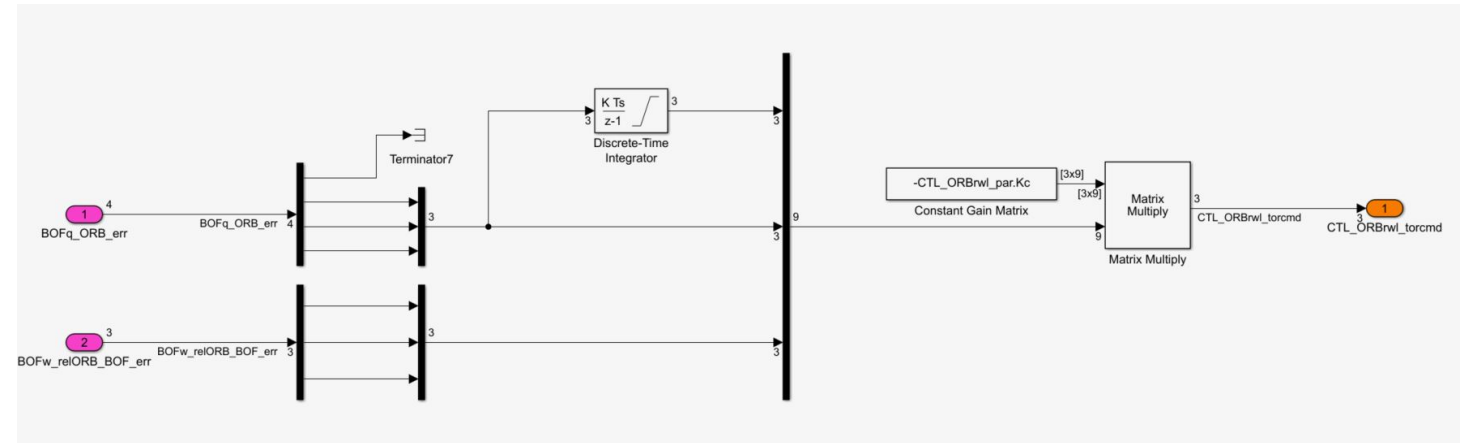

Figure 4.1.3: Controller Block

\section{$4.2 \quad$ Test Setup}

This section is to discuss how the test is set up and how the software is broken down. The test consists of a Monte Carlo simulation that randomizes the initial conditions and tests the controller gains throughout a large number of simulations. The test will run the simulation for 2 orbits and the errors will be observed after the initial settling time.The tolerances for both the Roll,Pitch, Yaw errors, 
and the angular rates errors were derived from the requirements document for the ESSENCE project, and can be summarized in table (4.1) [1]

Table 4.1: RPY and Rates Tolerances

\begin{tabular}{|c|c|}
\hline State Error & Tolerance \\
\hline RPY & $\pm 0.5 \mathrm{deg}$ \\
\hline Angular Rates & $\pm 0.05 \mathrm{deg} / \mathrm{s}$ \\
\hline
\end{tabular}

The full test can be broken down into multiple different MATLAB scripts to avoid having one large bundle of code. This makes troubleshooting and making changes to the code much easier, and it makes the code more modular:

1. runTST_CTL_ORBrwl_montecarlo.m

- This is the main script that runs the montecarlo simulation.

- Sets a clean start and initializes all directories for ESSENCE.

- Sets the correct directory and test folder

- Sets orbital duration (ie. number of orbits for test).

- Sets desired attitude states.

- Sets attitude and Rates Error tolerances.

- Builds SIMULINK model

- Calls other scripts and runs the simulation

2. TST_CTL_ORBrwl_monte_Controller.m

- Builds the augmented state-space equation.

- User manually sets the $\boldsymbol{R}$ and $\boldsymbol{Q}$ matrices for the LQR.

- Gain matrix $\boldsymbol{K}$ is calculated using lqr MATLAB function.

3. TST_CTL_ORBrwl_monte_init.m

- Randomizes center of mass distribution

- Randomizes initial attitude states within a 360 degree sweep, between \pm 180 deg. 
- Randomizes initial rates between $\pm 0.02 \mathrm{deg} / \mathrm{s}$

- Sets the inputs needed for the simulation in a SimIn set.

4. TST_CTL_ORBrwl_monte_CheckandPlot.m

- Sets the result folder.

- Checks the RPY and Rates signal error values and compares to tolerances set in the main run script.

- If both checks pass then it generates plots for initial values.

- If either of them fail the check then more plots summarizing the full attitude summary is plotted to give more information on what failed and why.

5. plotTST_CTL_ORBrwl_monte.m

- A plot function used in the TST_CTL_ORBrwl_monte_CheckandPlot.m script.

- Takes outputs from the simulation and generates plots.

- function is written so that user can chose which set of plots to generate.

6. TST_CTL_ORBrwl_monte_Stats.m

- Plots the main statistics of the full simulation set

- Initial RPY and rates for each simulation.

- Average errors for each simulation.

- Maximum and minimum command torques experienced by the wheels.

- Maximum and minimum wheel speed, to ensure wheels are not oversaturated.

All the other scripts are called into the main run script. The flow of the software can be shown in figure (4.2.1) 


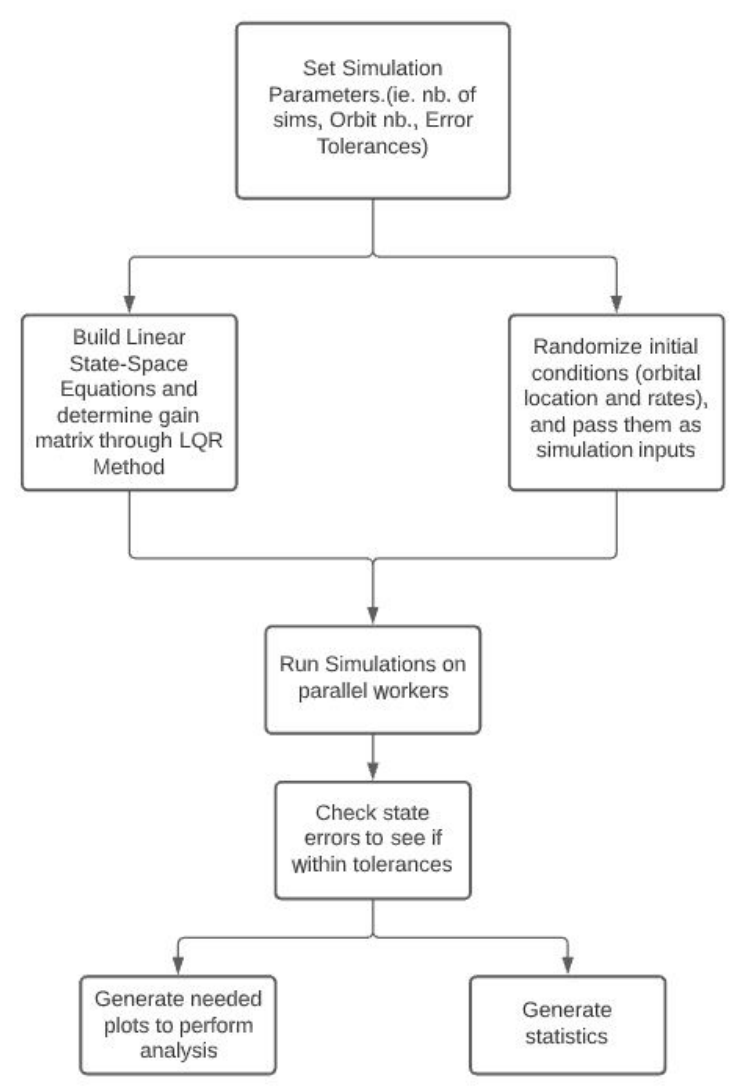

Figure 4.2.1: Software Flowchart

\subsection{Results}

The testing done in this project has been rather robust. Five controllers have been tested for 200 simulations each, with completely randomized initial conditions. Each of the following sections includes a table that summarizes the success rate of the gain matrix used, along with the $\mathrm{Q}$ and $\mathrm{R}$ matrices that were used to compute that gain matrix. After that, a set of figures is displayed, showing the randomization in each simulation, the average error for each simulation, the maximum and minimum control torque input, and the maximum and minimum reaction wheel speeds. 


\subsubsection{Simulation Set 1}

Table 4.2: Summary of Results for Simulation Set 1

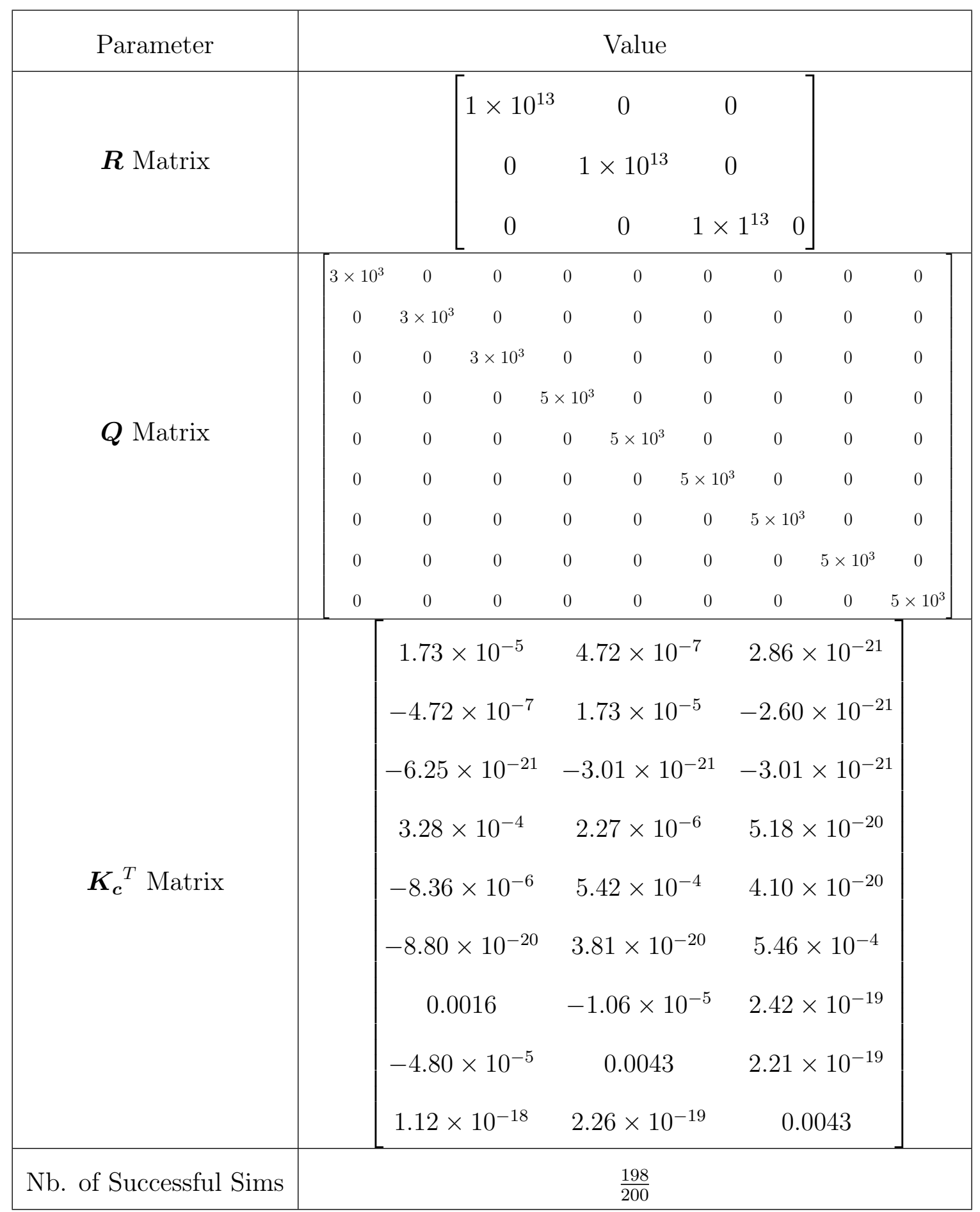




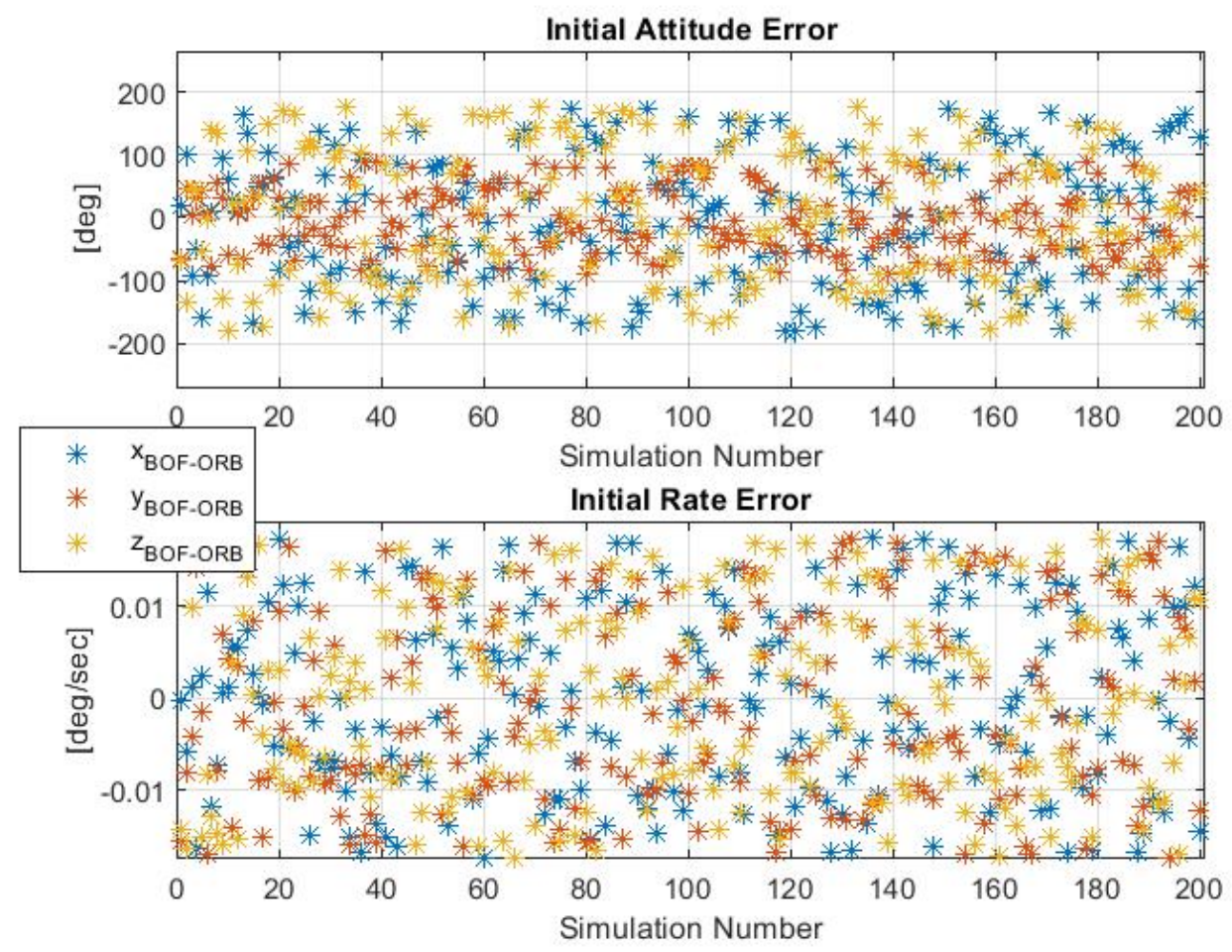

Figure 4.3.1: Initial Errors for Each Simulation - Set 1

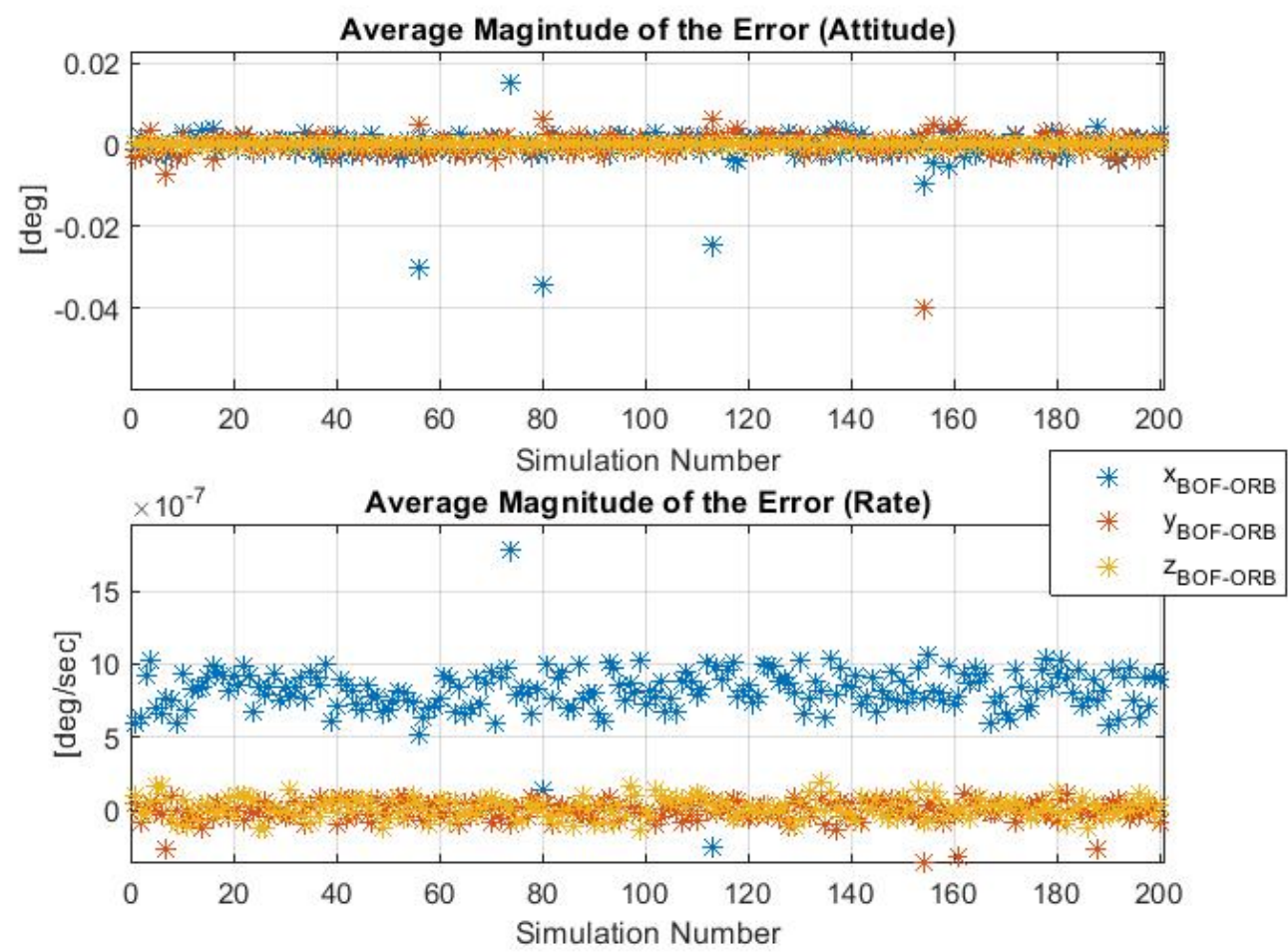

Figure 4.3.2: Average Errors for Each Simulation - Set 1 

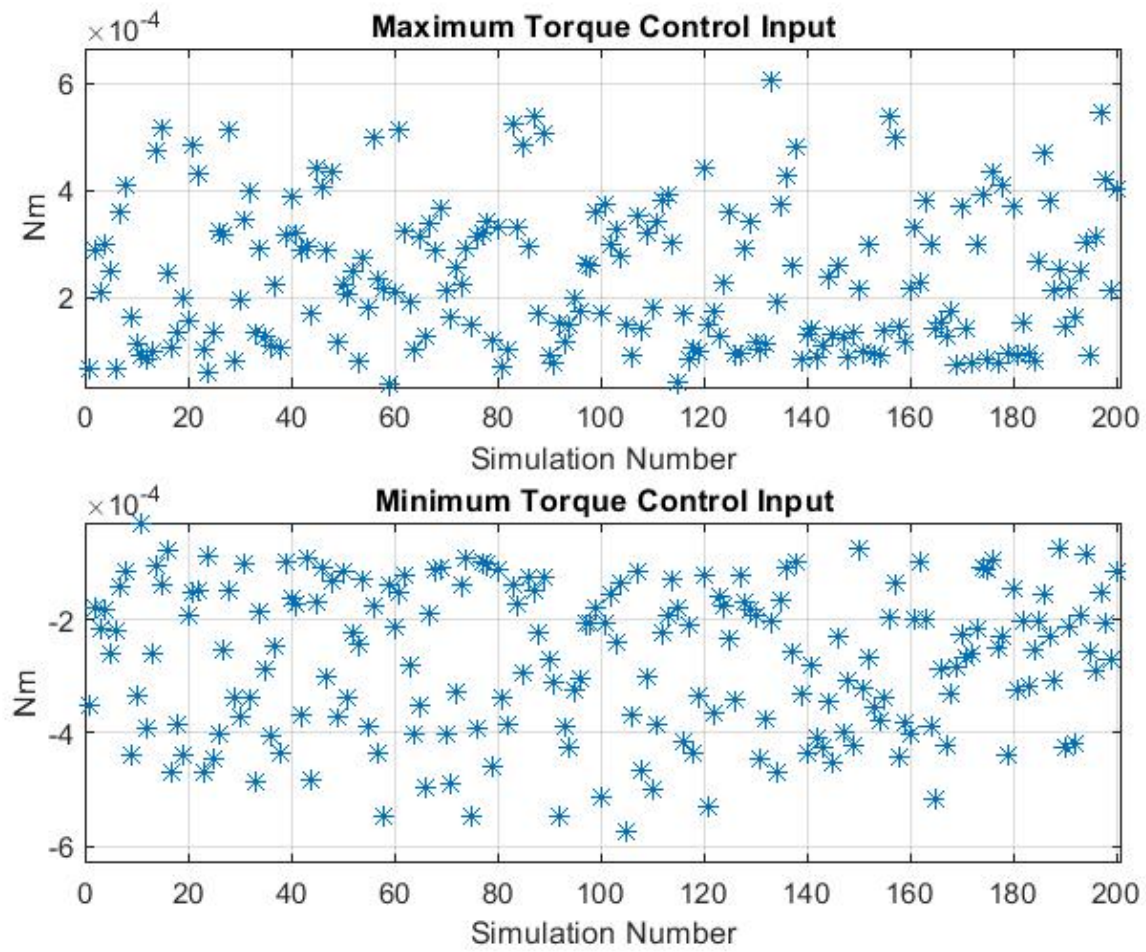

Figure 4.3.3: Maximum Control Inputs - Set 1
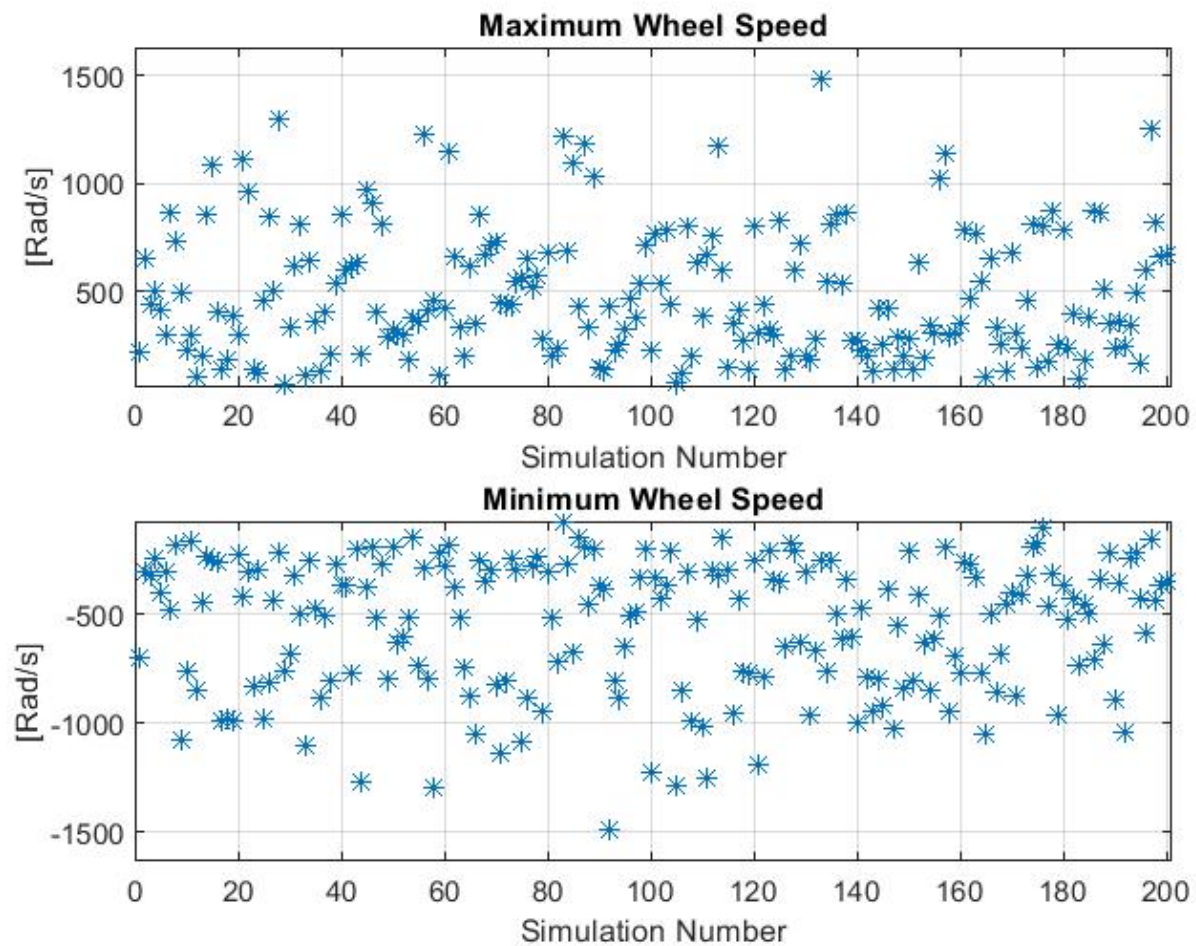

Figure 4.3.4: Maximum and Minimum Reaction Wheel Speed - Set 1 

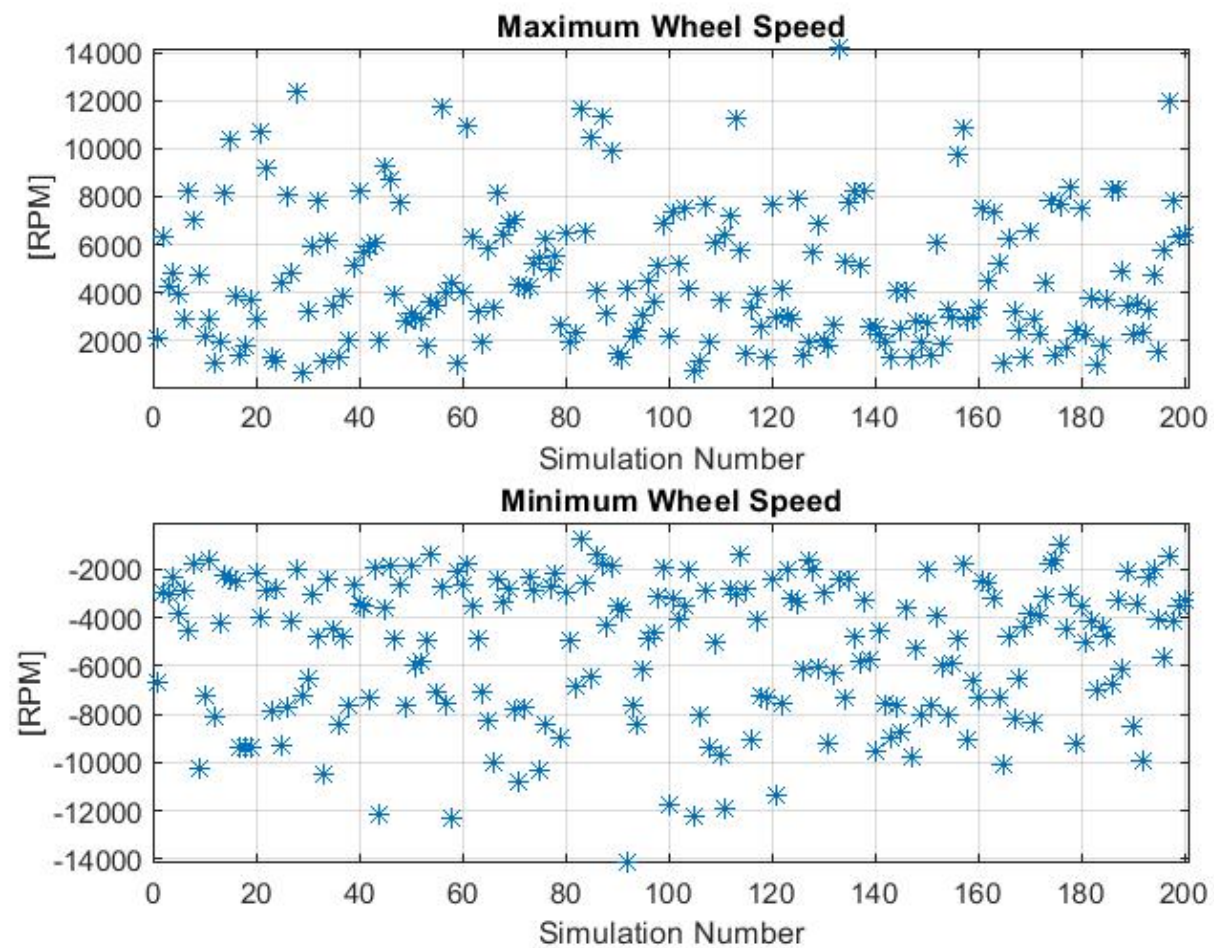

Figure 4.3.5: Maximum and Minimum Reaction Wheel Speed [RPM]- Set 1

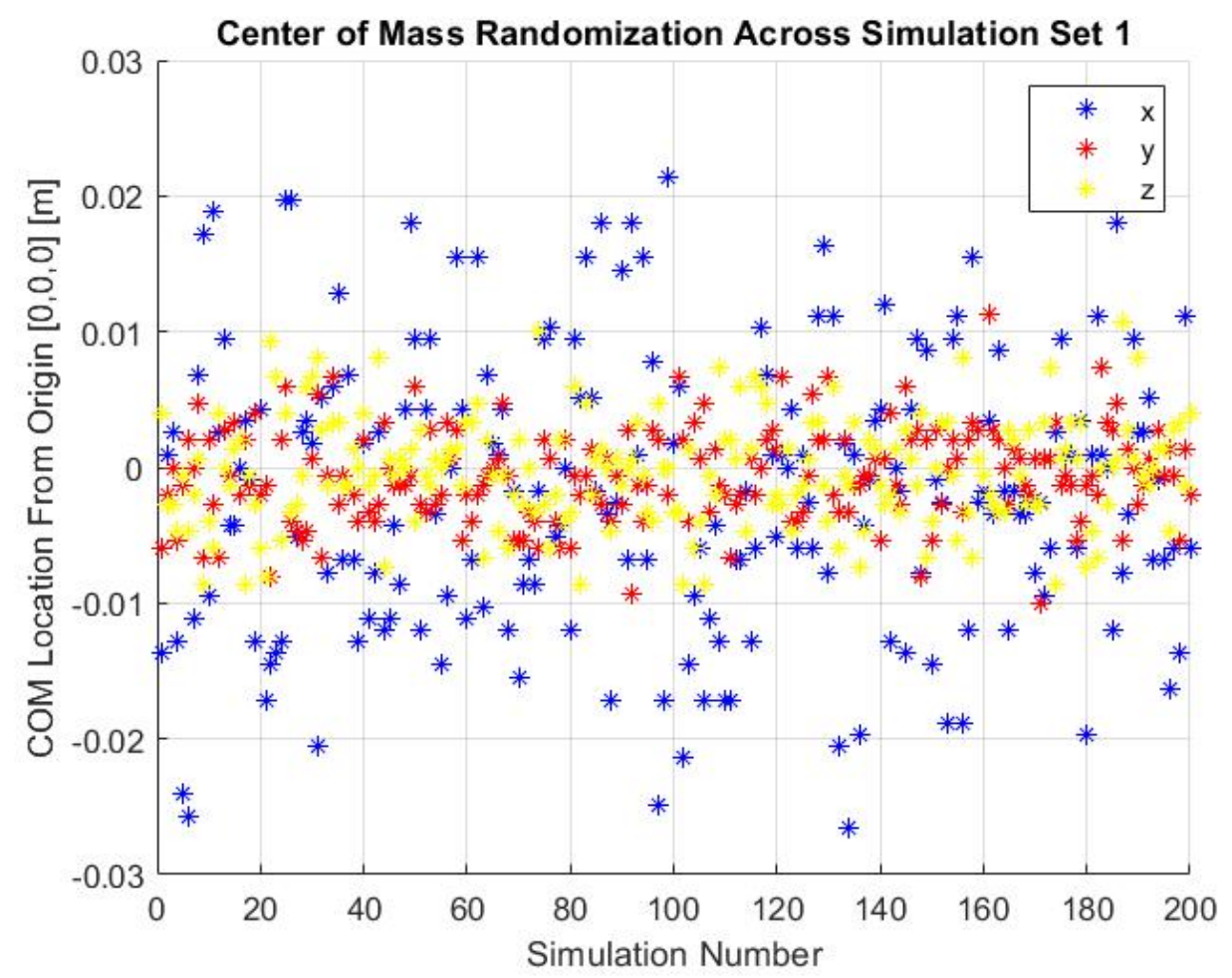

Figure 4.3.6: Center of Mass Distribution - Set 1 


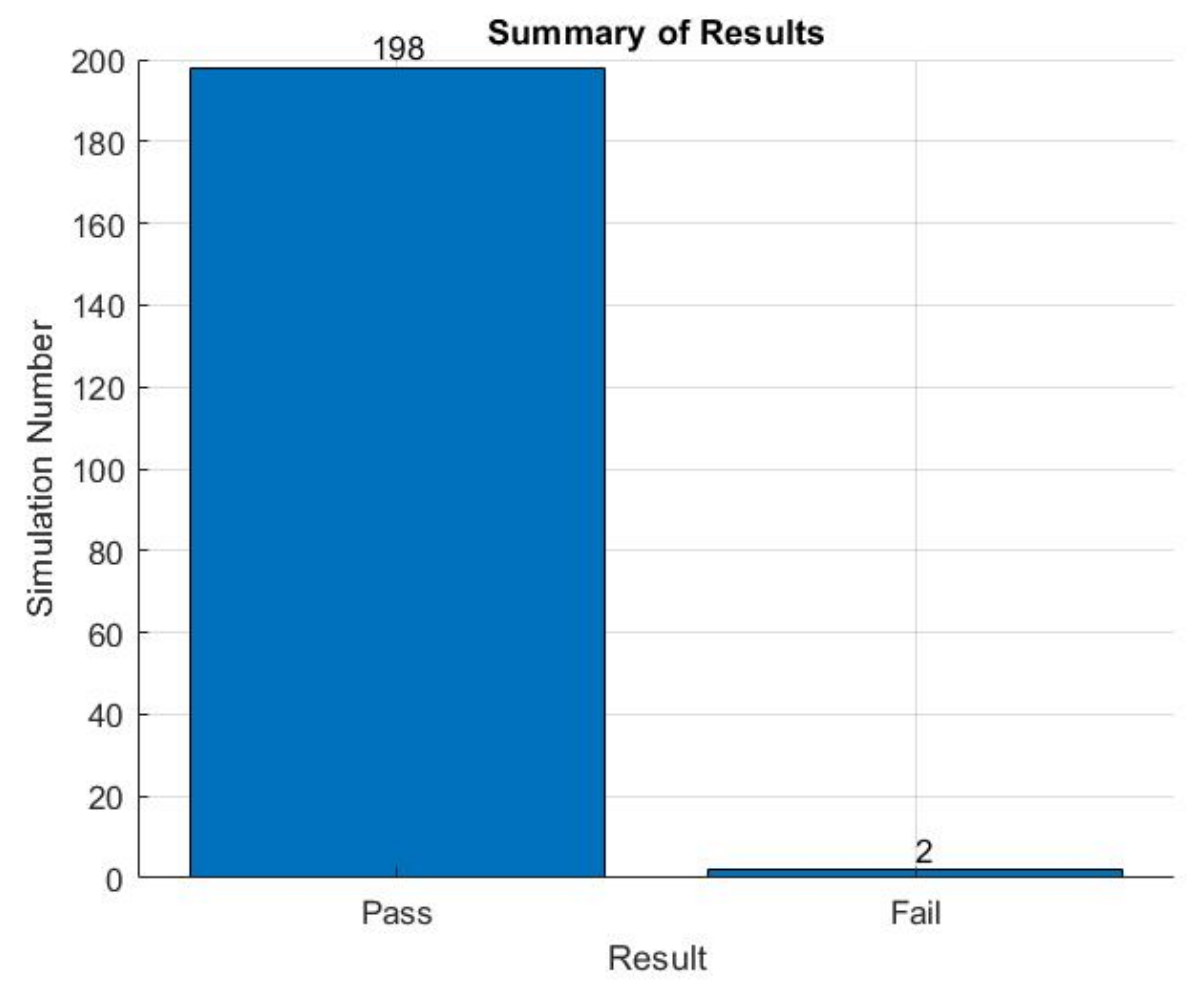

Figure 4.3.7: Summary of Results - Set 1

Although the RPY and angular rate conditions were met for all the simulations in this set, the reason two simulations were classified as "Fails" is because the reaction wheels were over-saturated. This can be seen in figure (4.3.4), where the reaction wheel speed for both simulations 92 and 133 reaches the reaction wheel limit of $1484 \mathrm{rad} / \mathrm{sec}$. Typically, this issue can be resolved by using magnetorquers and momentum dumping, but for the sake of this report, any test that results in the over-saturation of the reaction wheels, the test will be classified as a "Fail" 


\subsubsection{Simulation Set 2}

Table 4.3: Summary of Results for Simulation Set 2

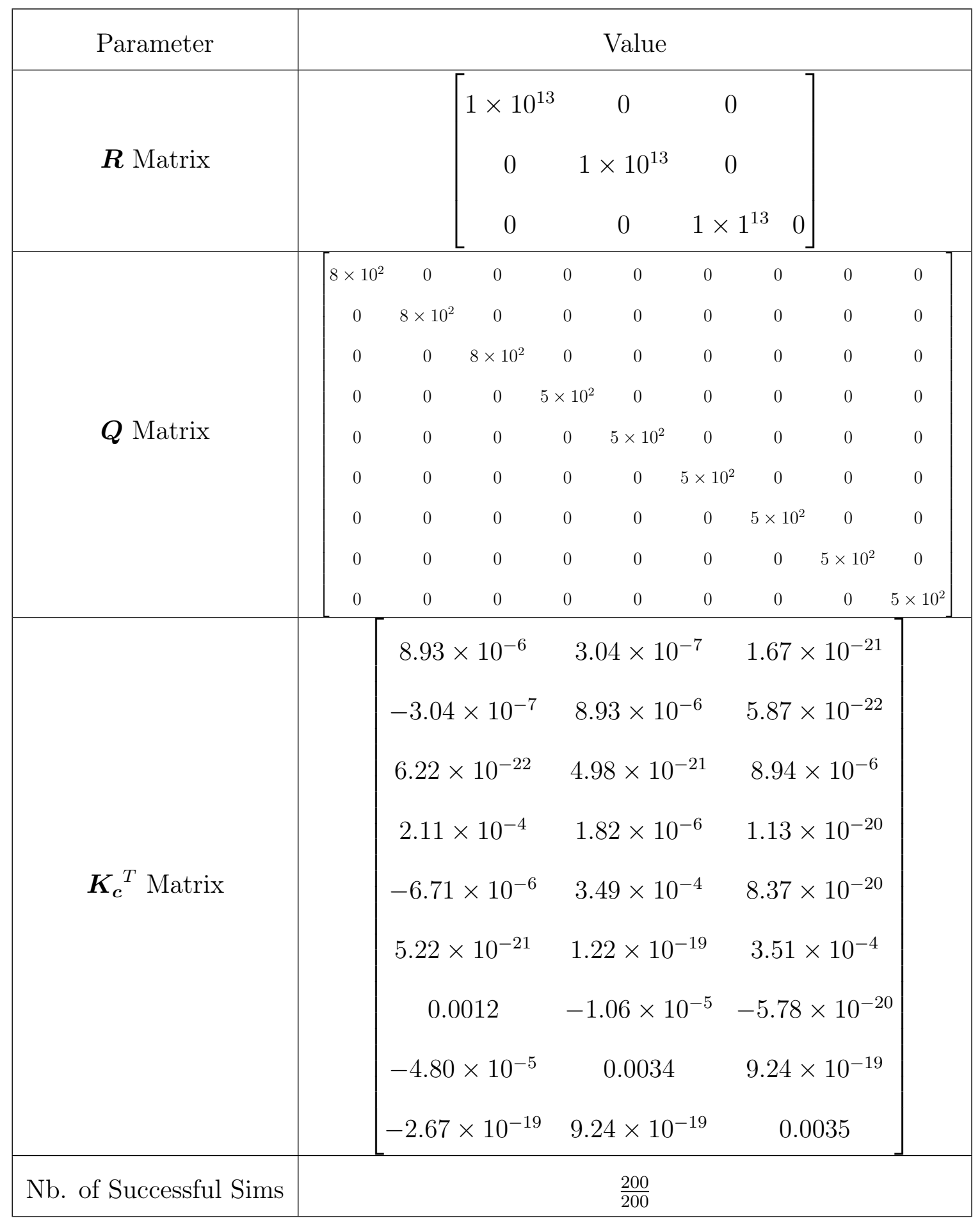




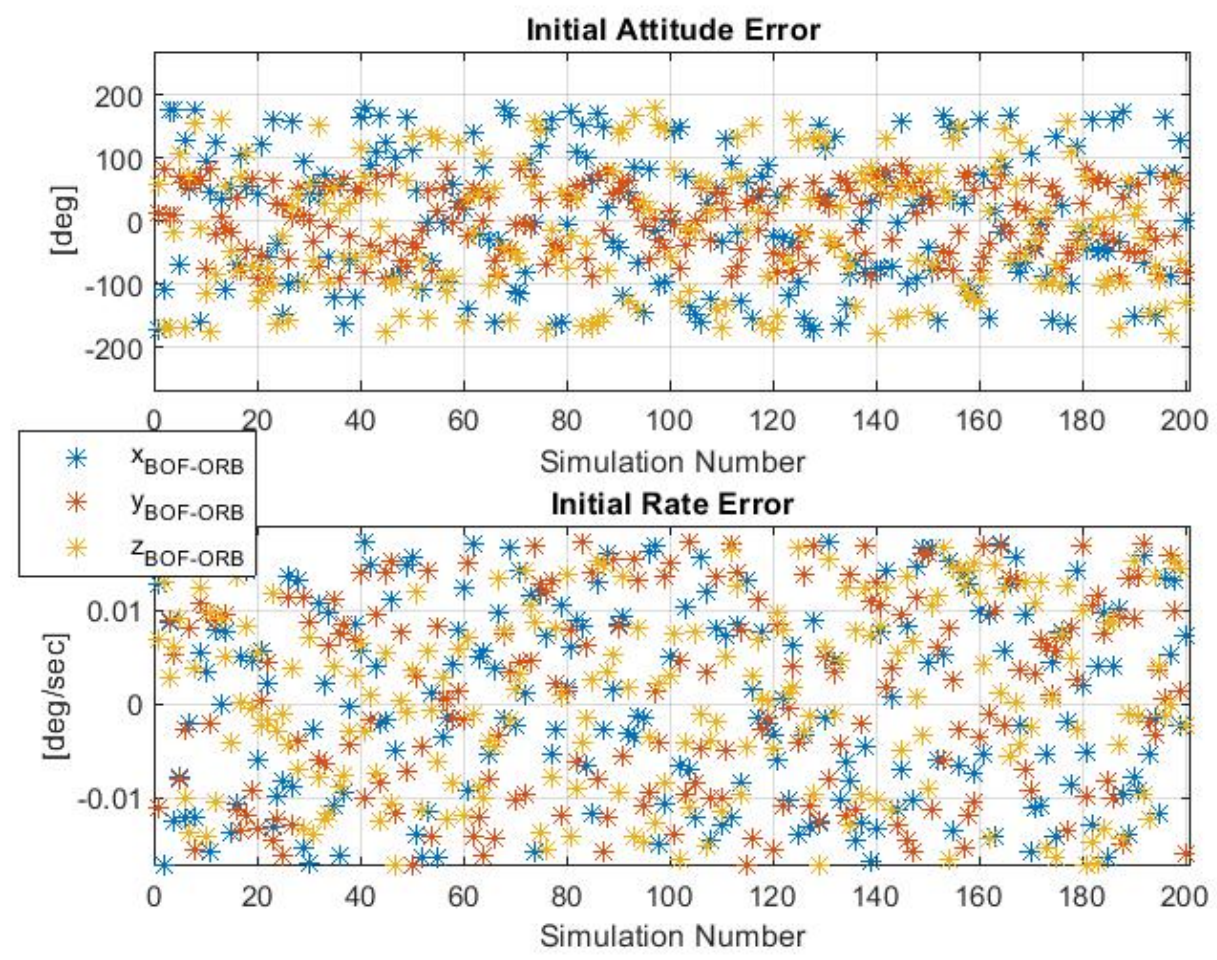

Figure 4.3.8: Initial Errors for Each Simulation - Set 2

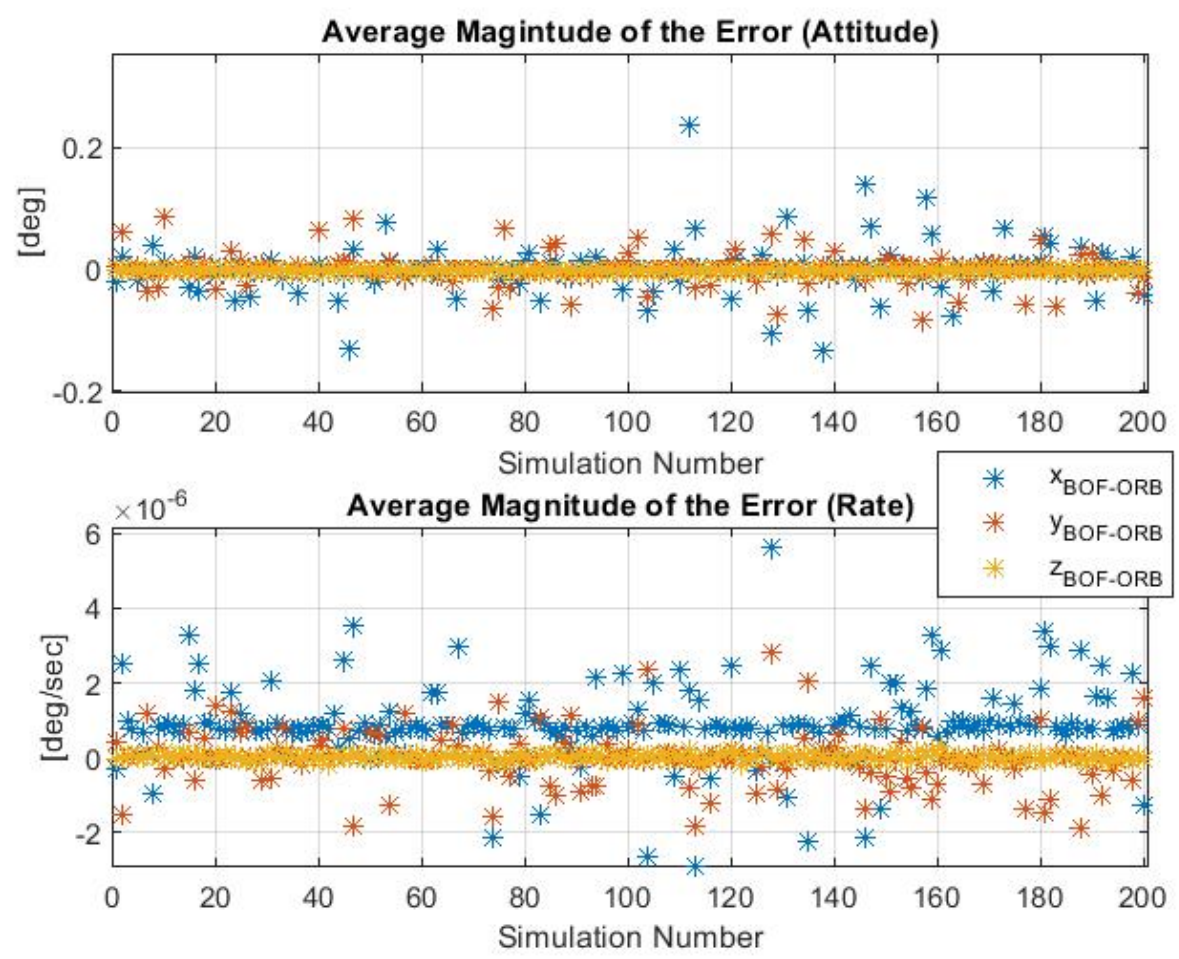

Figure 4.3.9: Average Errors for Each Simulation - Set 2 

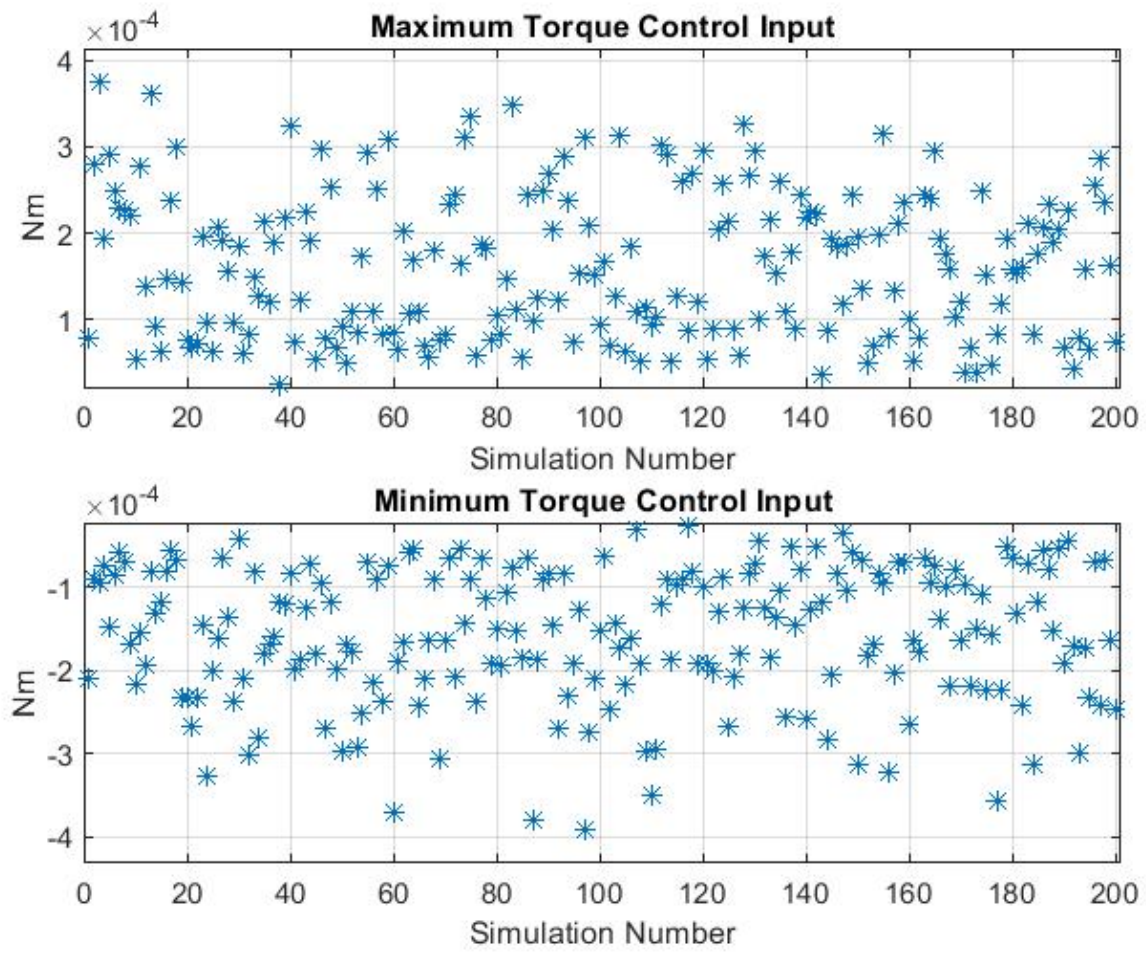

Figure 4.3.10: Maximum Control Inputs - Set 2
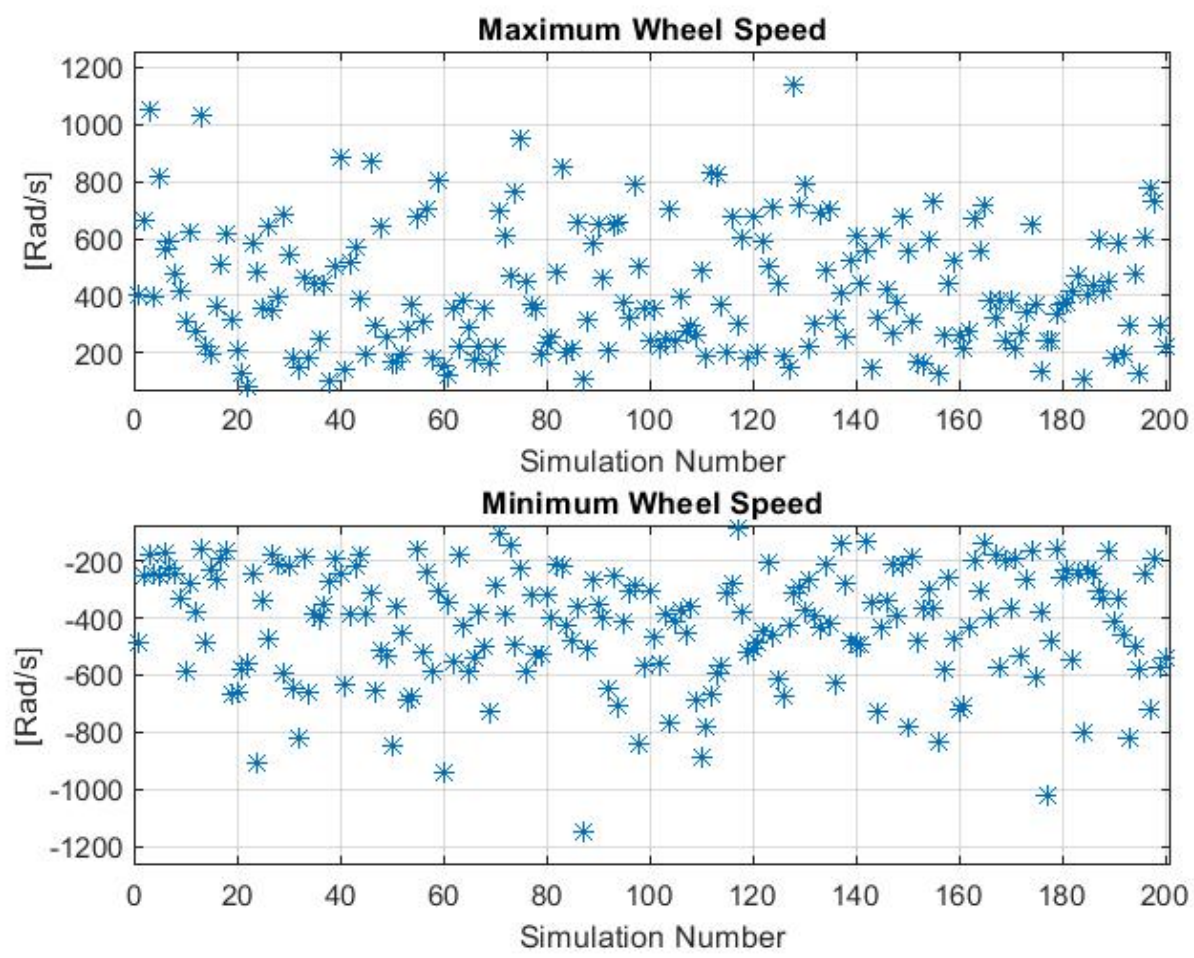

Figure 4.3.11: Maximum and Minimum Reaction Wheel Speed - Set 2 

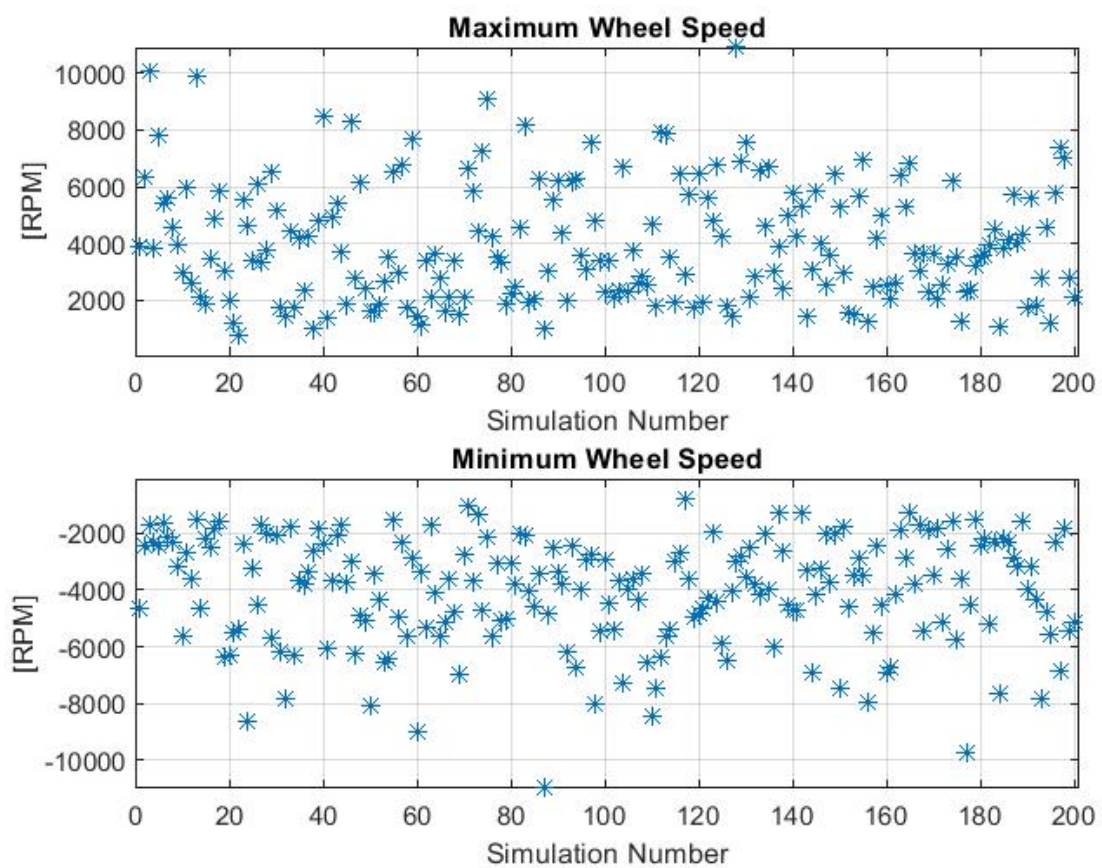

Figure 4.3.12: Maximum and Minimum Reaction Wheel Speed [RPM]- Set 2

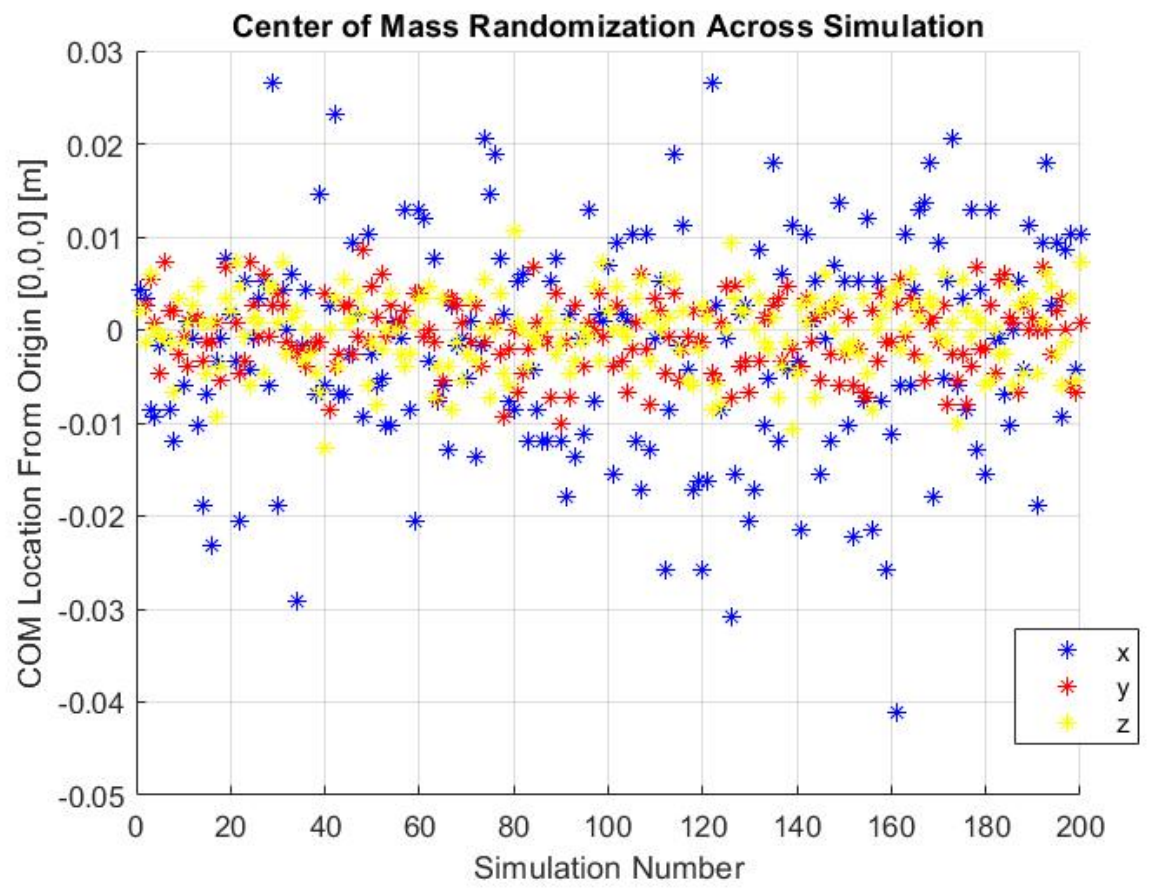

Figure 4.3.13: Center of Mass Distribution - Set 2 


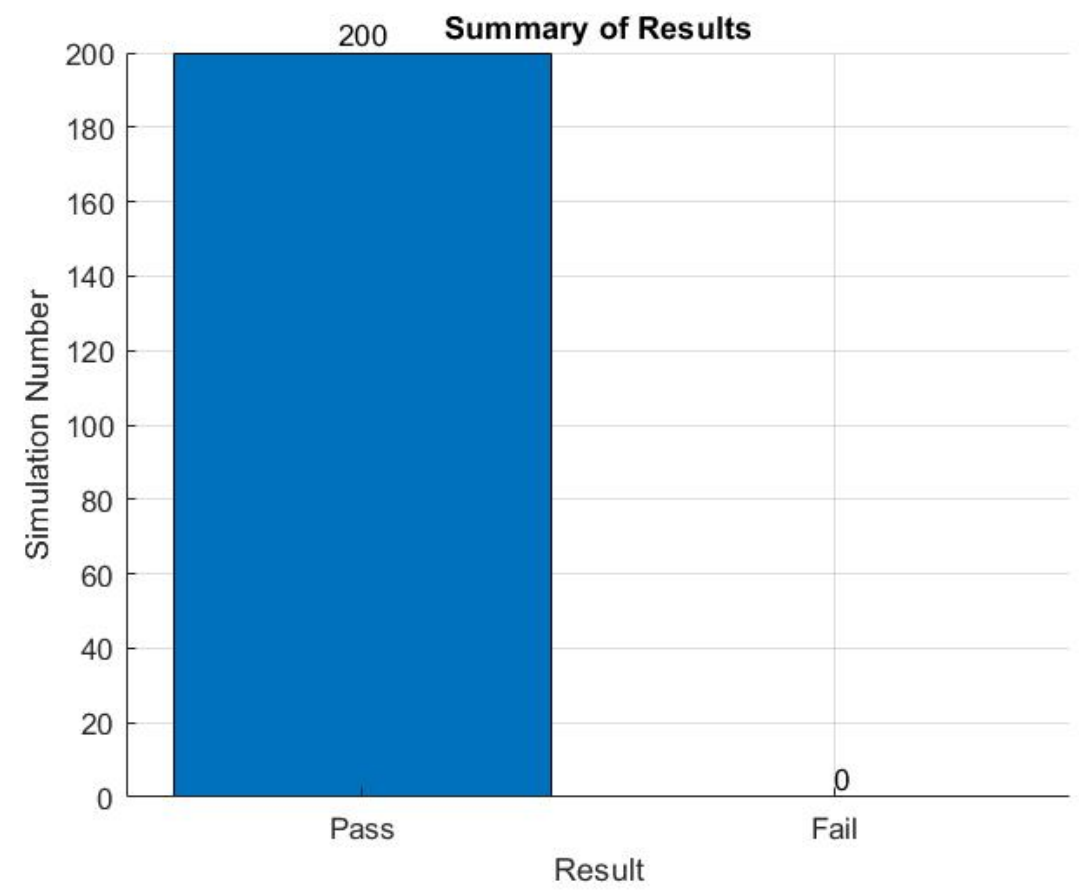

Figure 4.3.14: Summary of Results - Set 2 


\subsubsection{Simulation Set 3}

Table 4.4: Summary of Results for Simulation Set 3

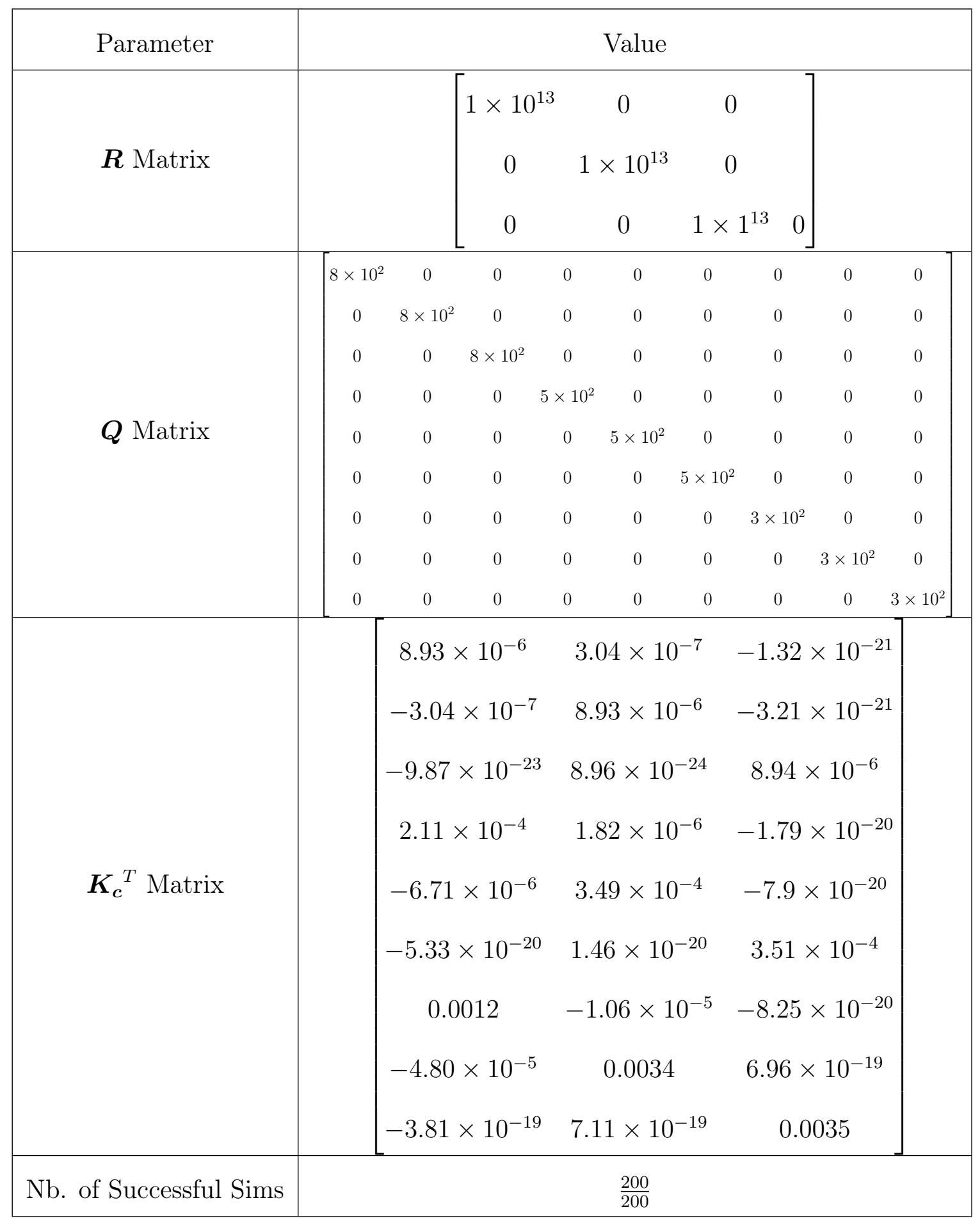




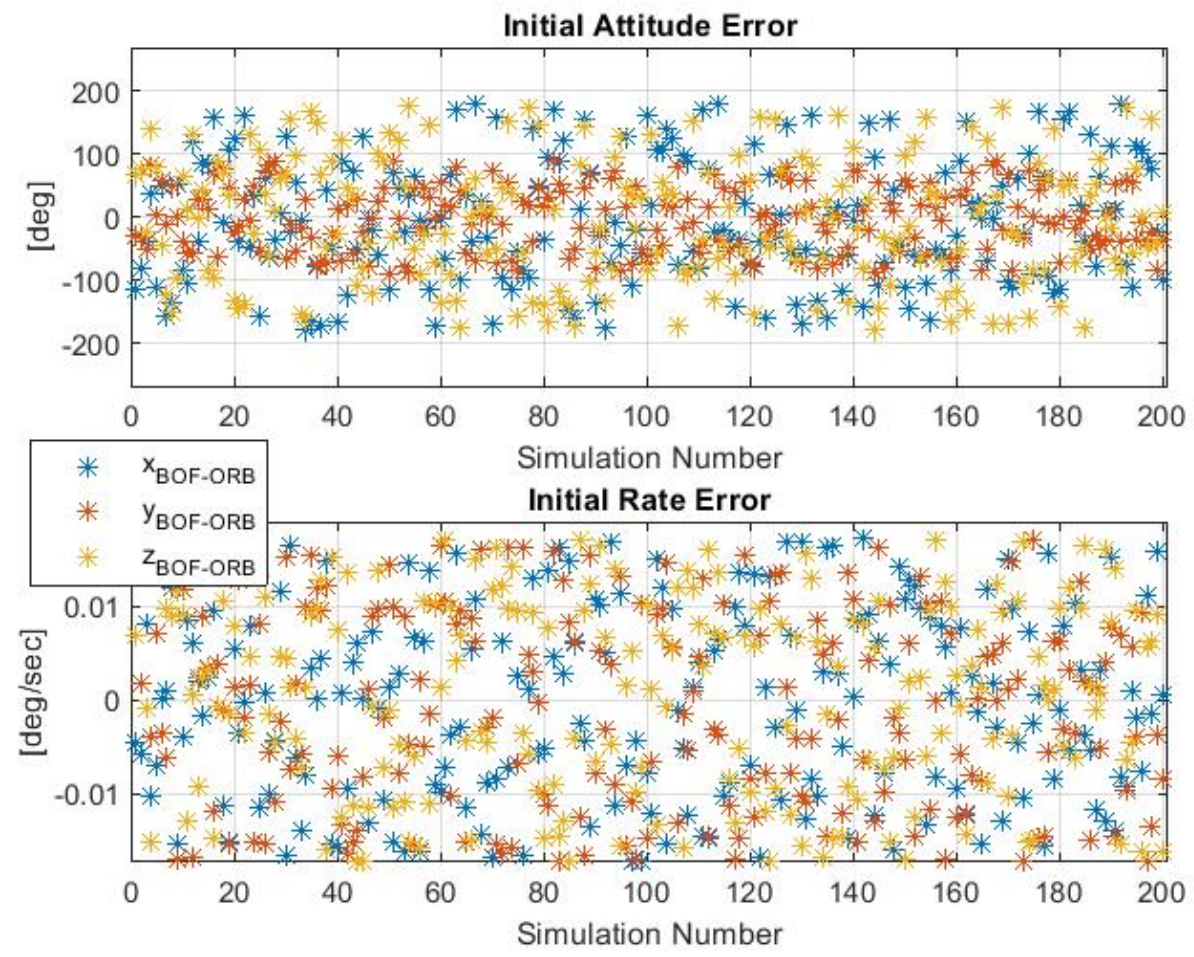

Figure 4.3.15: Initial Errors for Each Simulation - Set 3

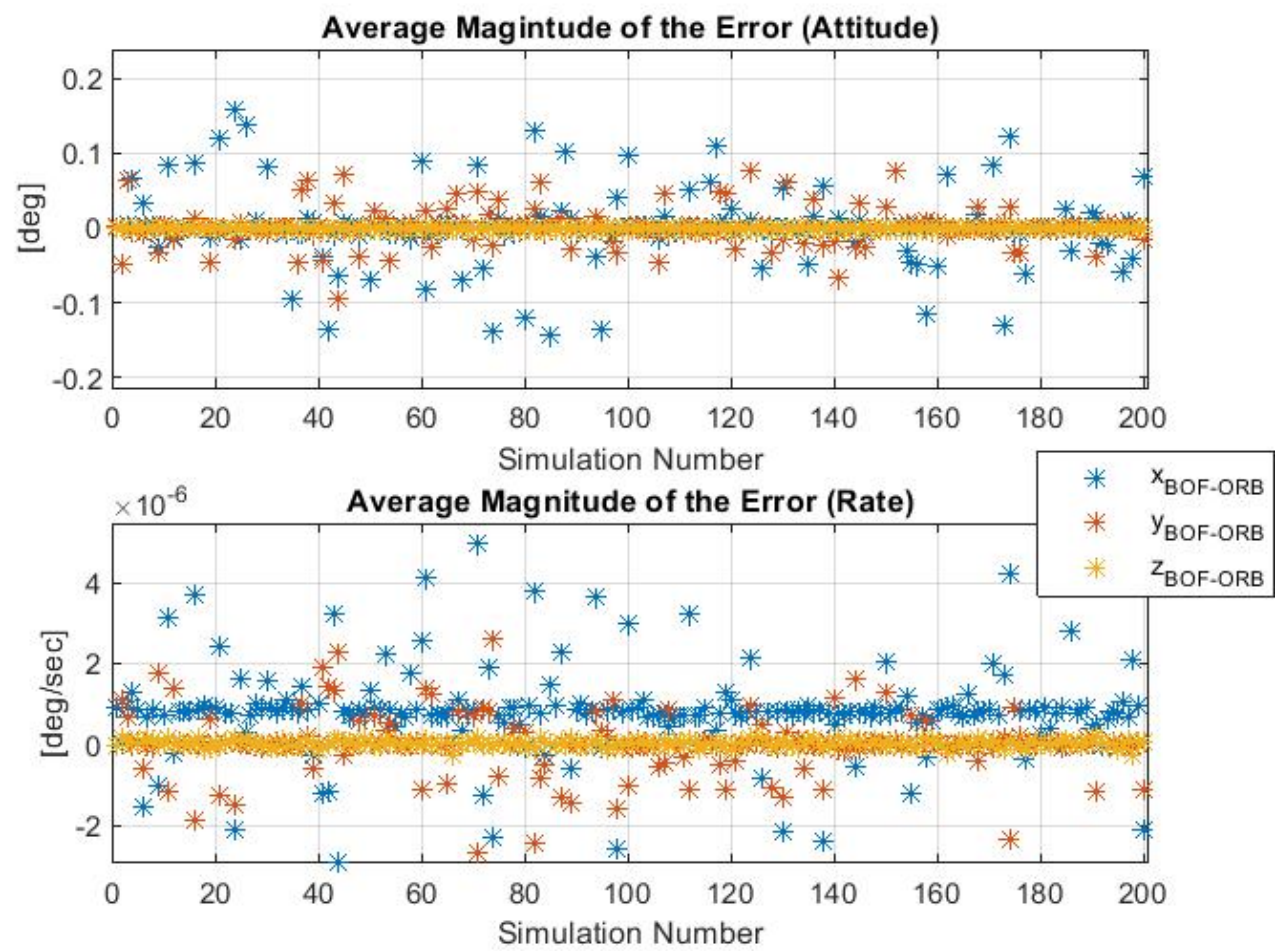

Figure 4.3.16: Average Errors for Each Simulation - Set 3 

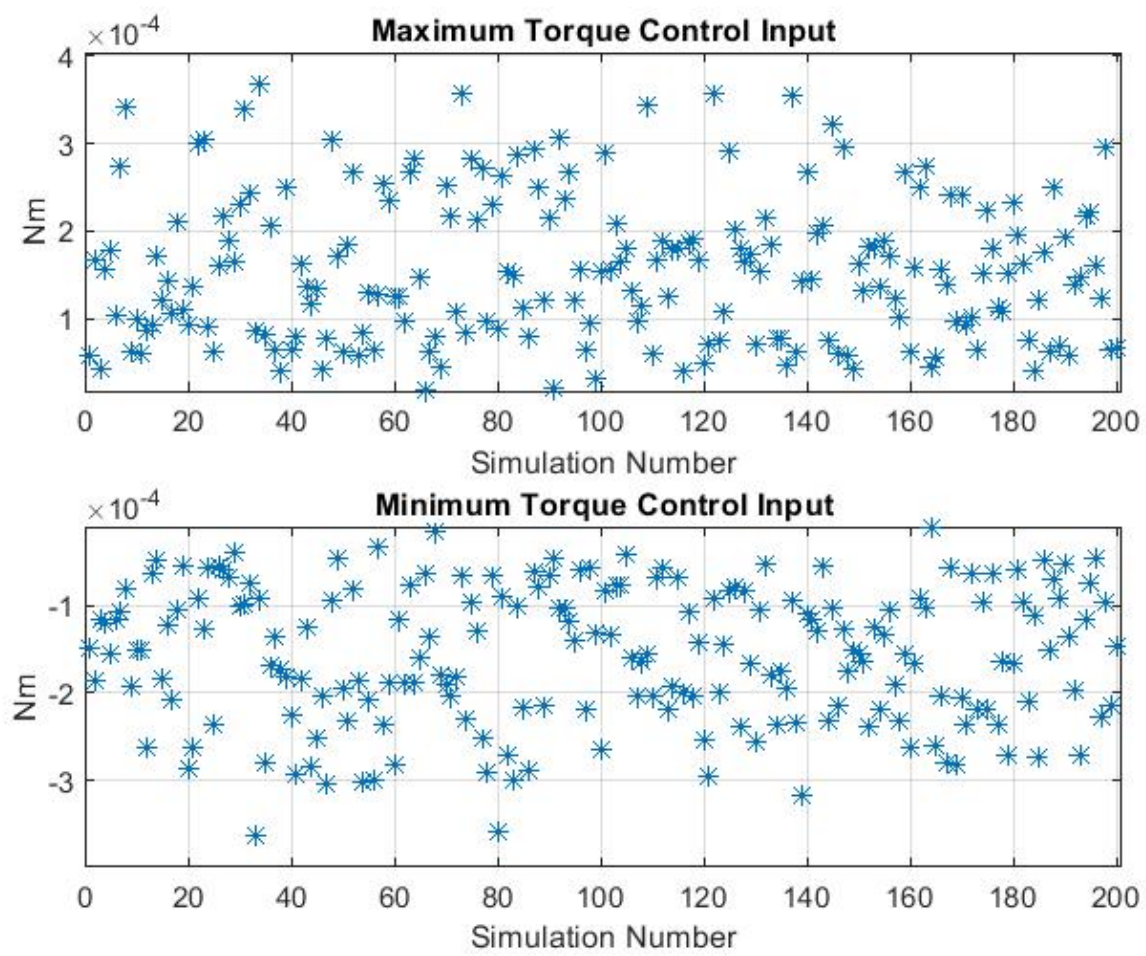

Figure 4.3.17: Maximum Control Inputs - Set 3

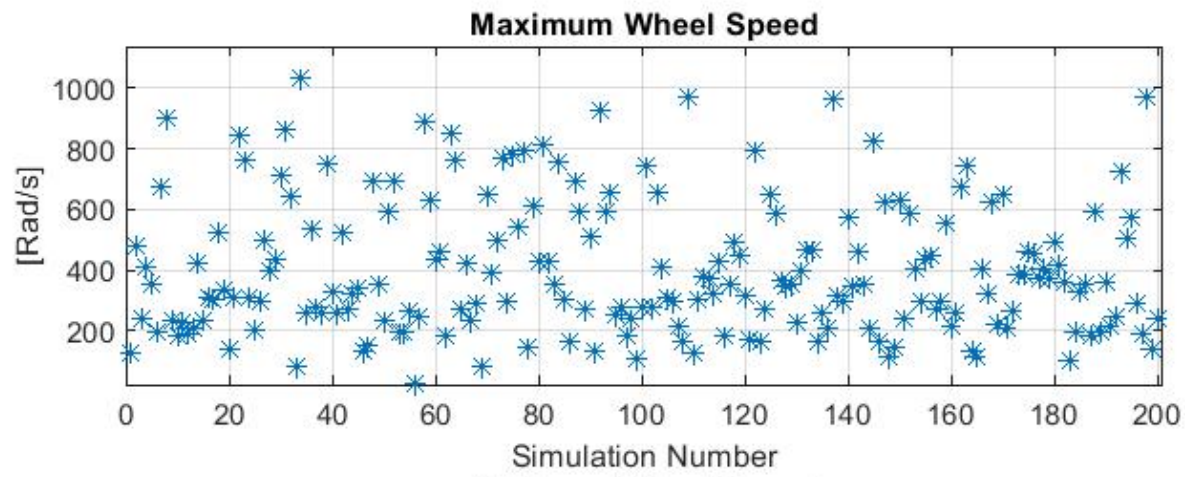

Minimum Wheel Speed

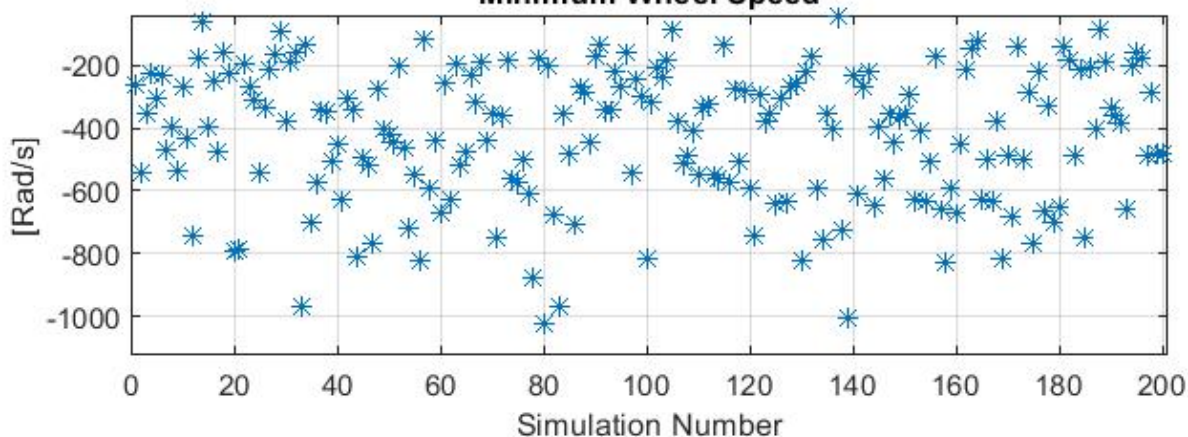

Figure 4.3.18: Maximum and Minimum Reaction Wheel Speed - Set 3 

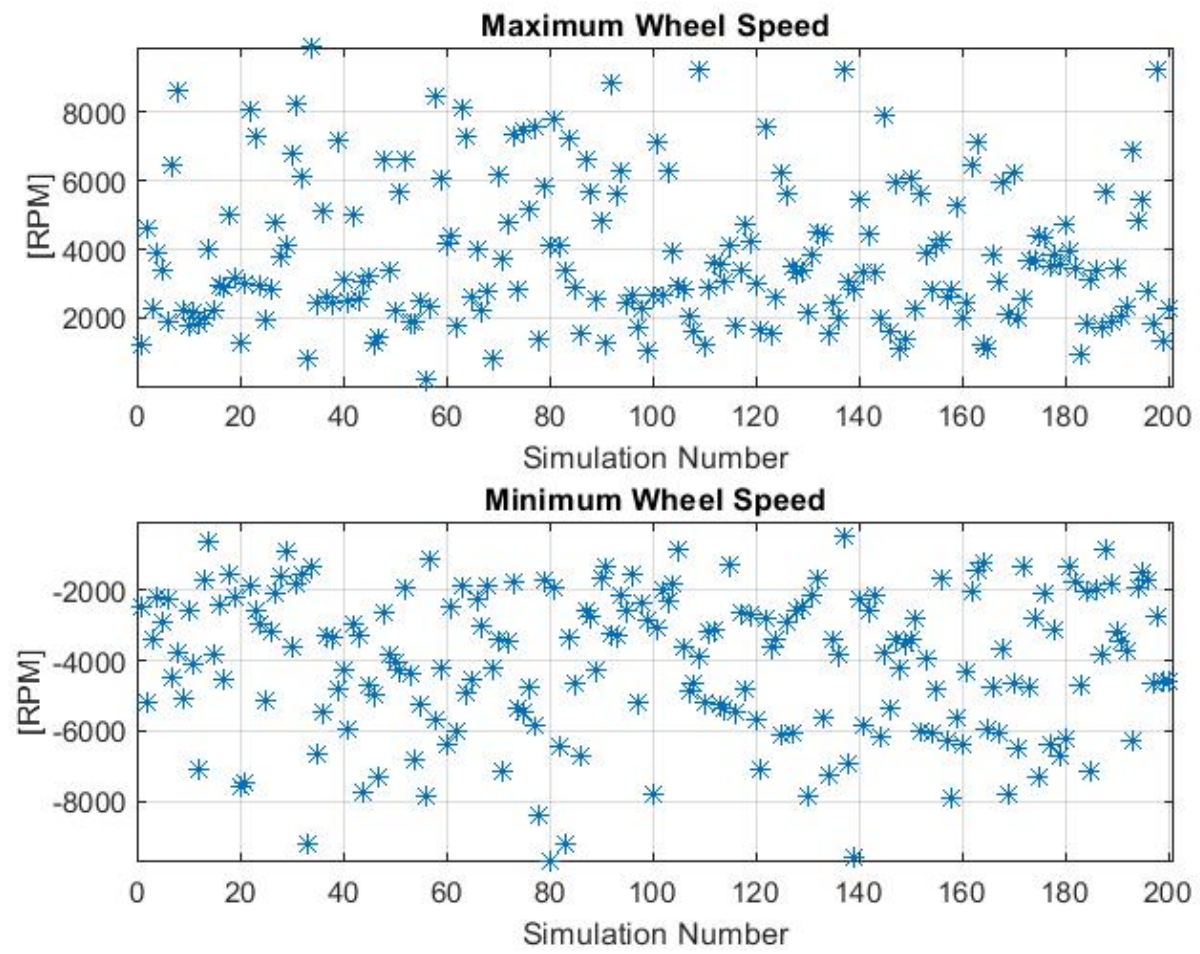

Figure 4.3.19: Maximum and Minimum Reaction Wheel Speed [RPM]- Set 3

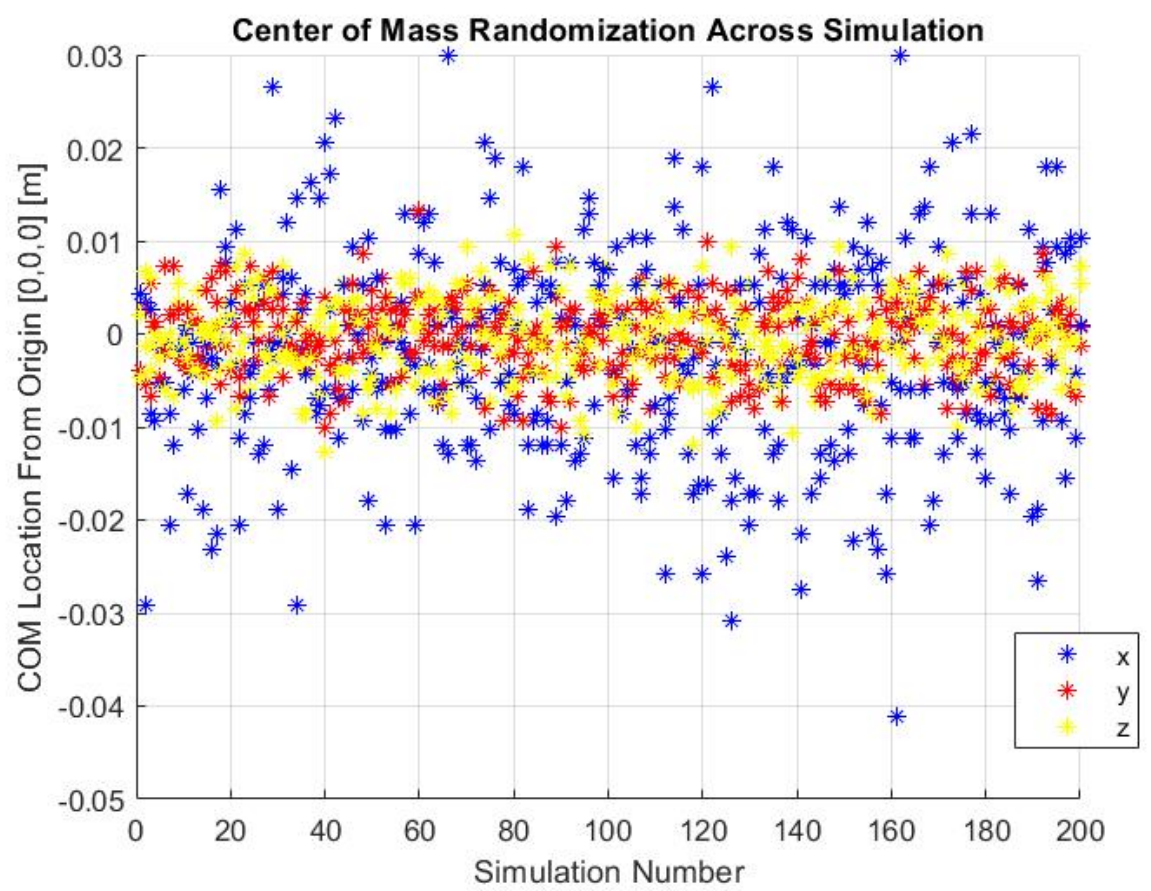

Figure 4.3.20: Center of Mass Distribution - Set 3 


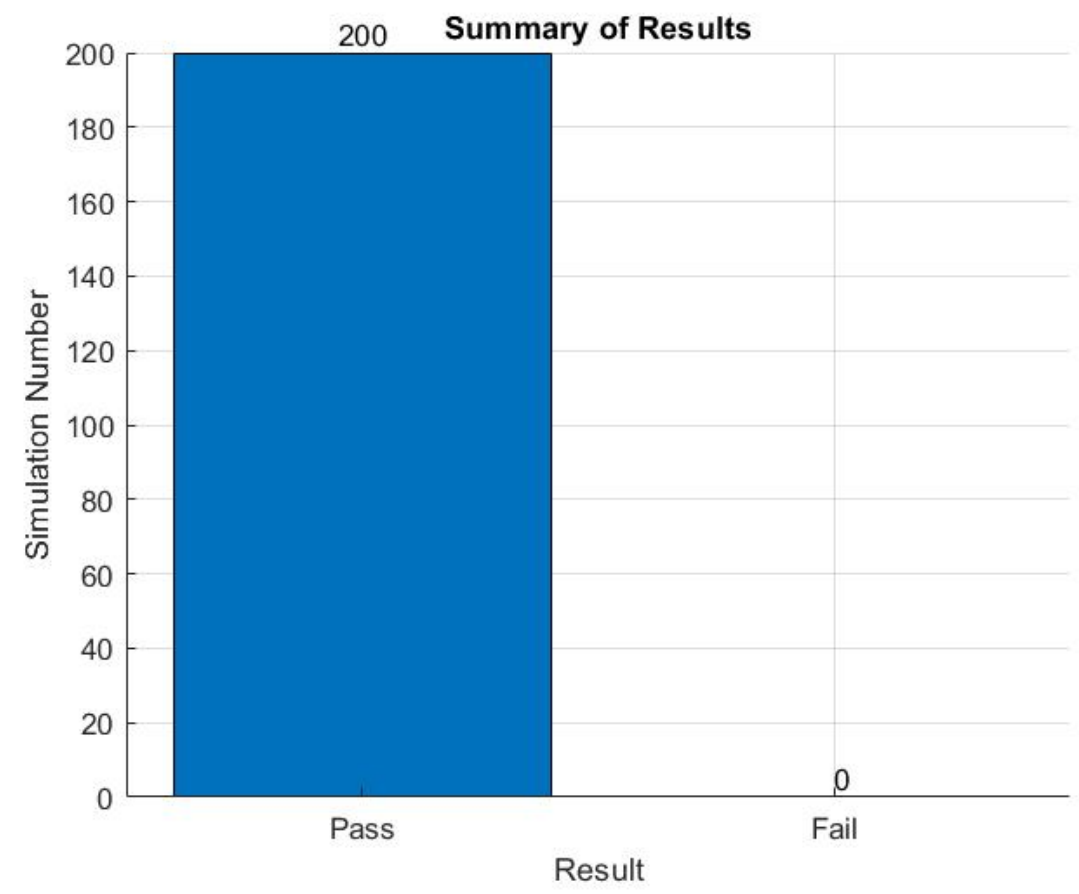

Figure 4.3.21: Summary of Results - Set 3 


\subsubsection{Simulation Set 4}

Table 4.5: Summary of Results for Simulation Set 4

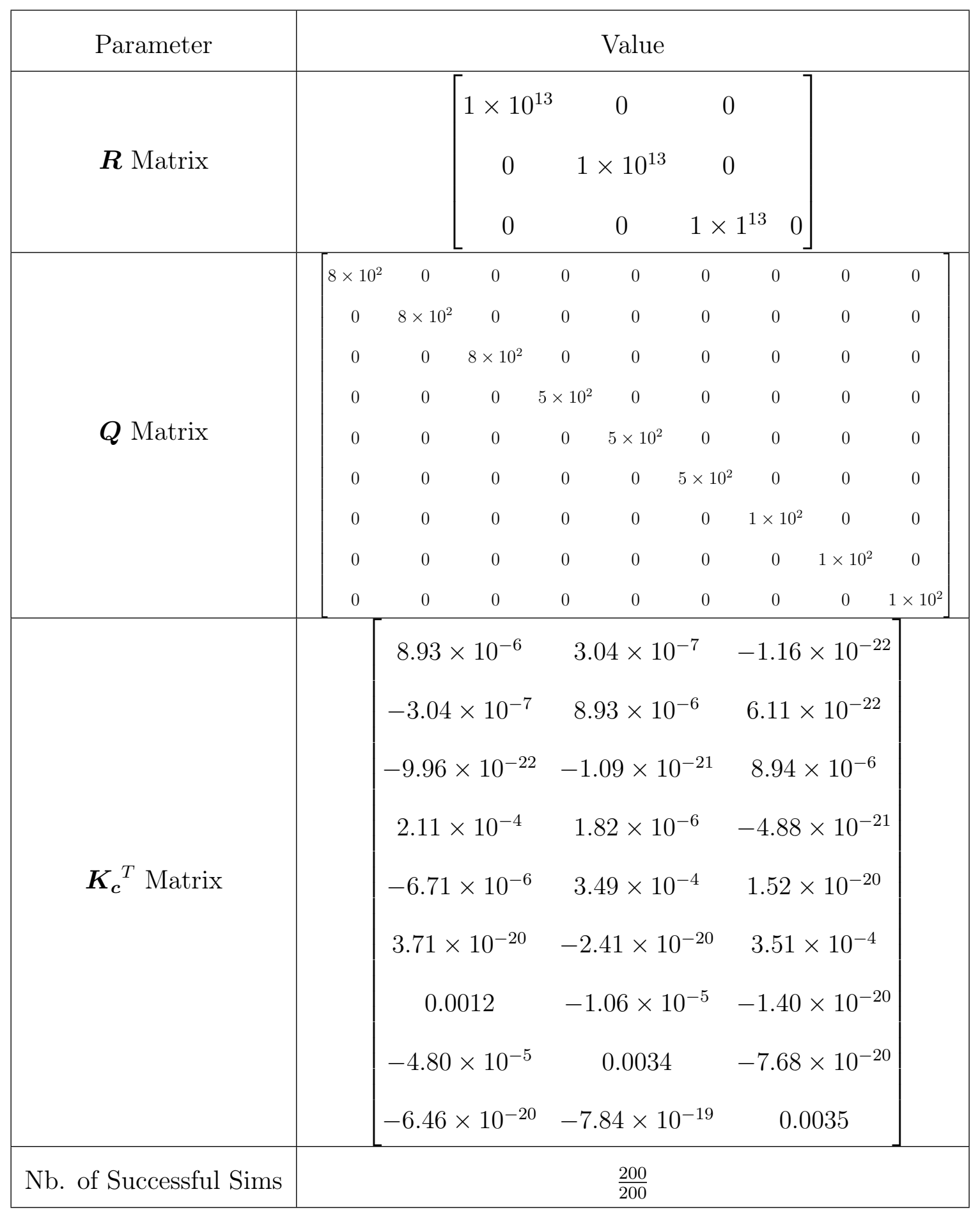




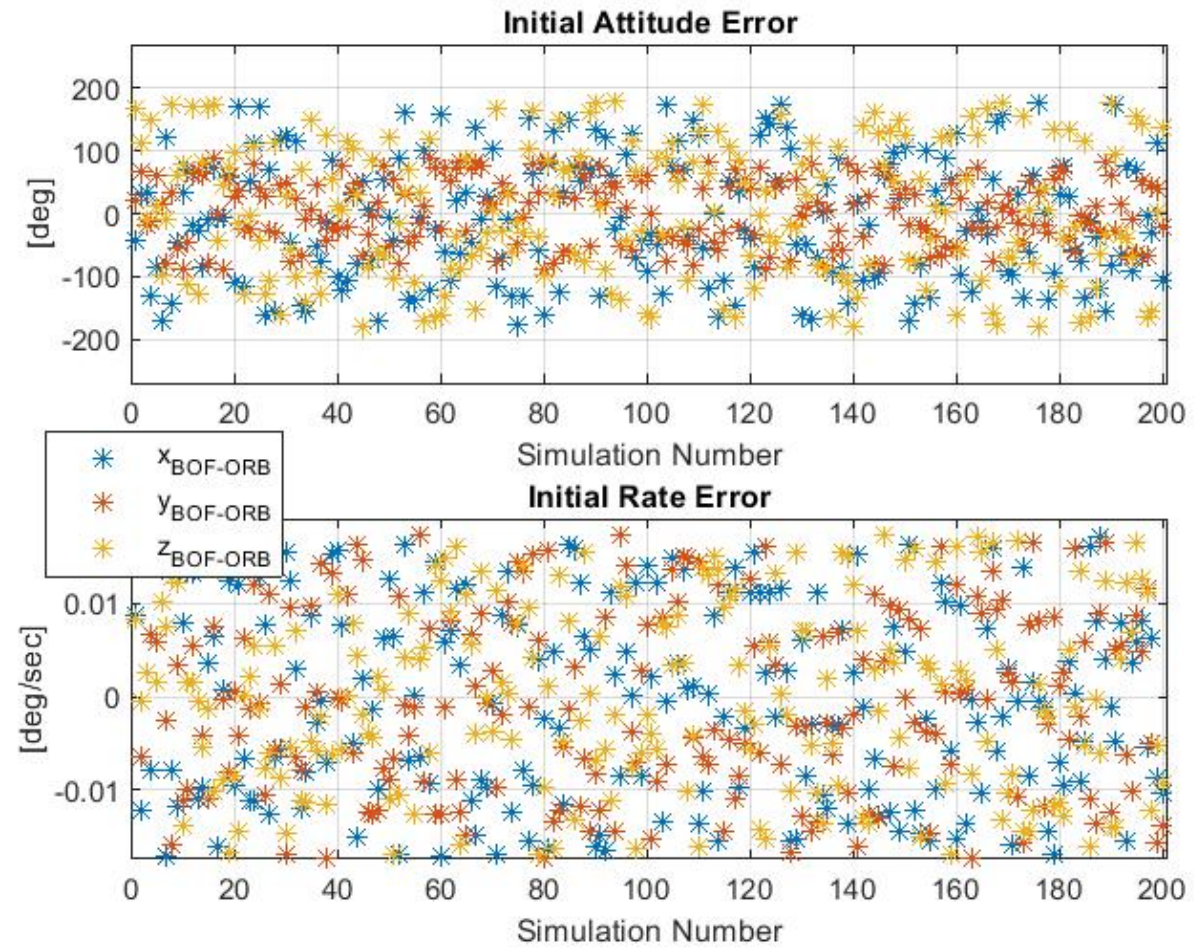

Figure 4.3.22: Initial Errors for Each Simulation - Set 4

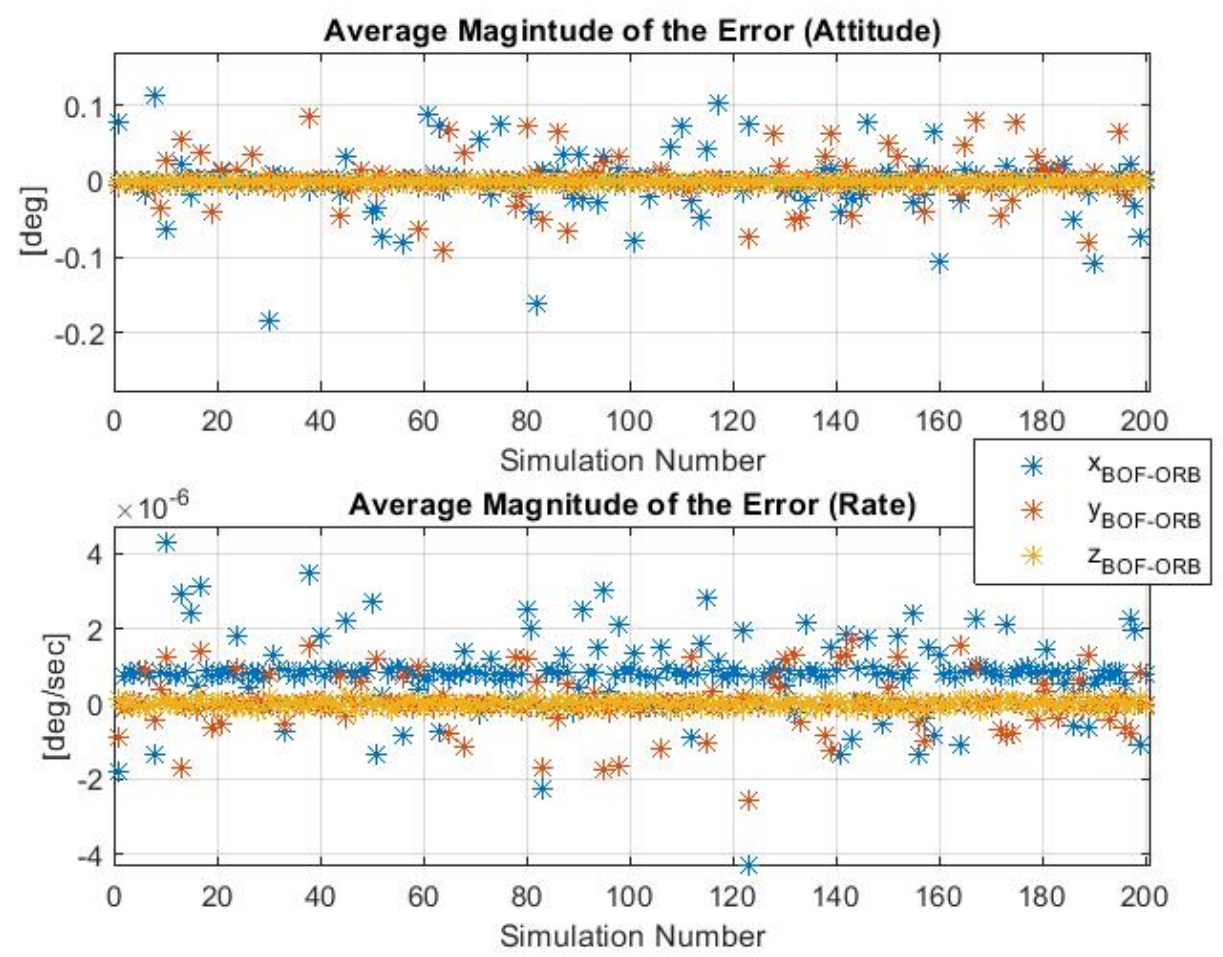

Figure 4.3.23: Average Errors for Each Simulation - Set 4 

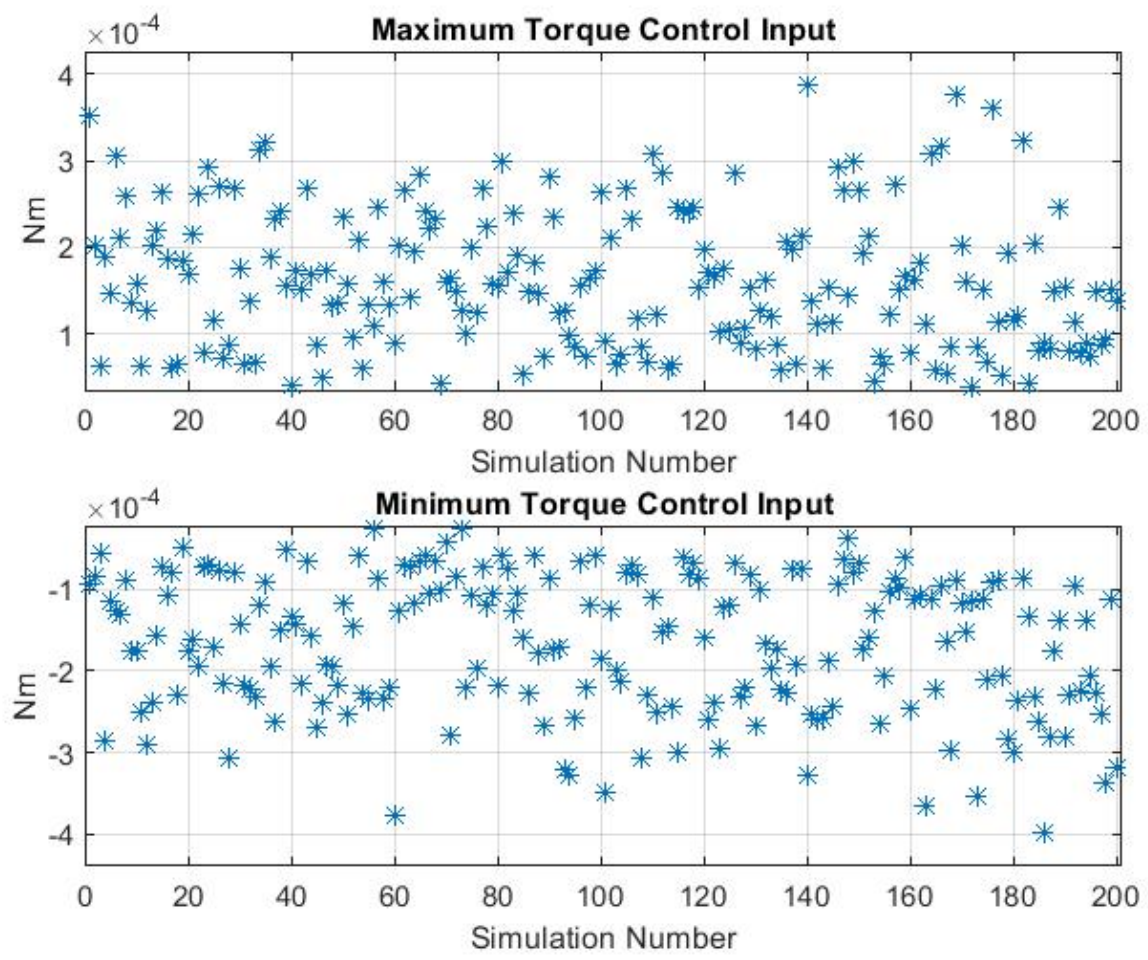

Figure 4.3.24: Maximum Control Inputs - Set 4

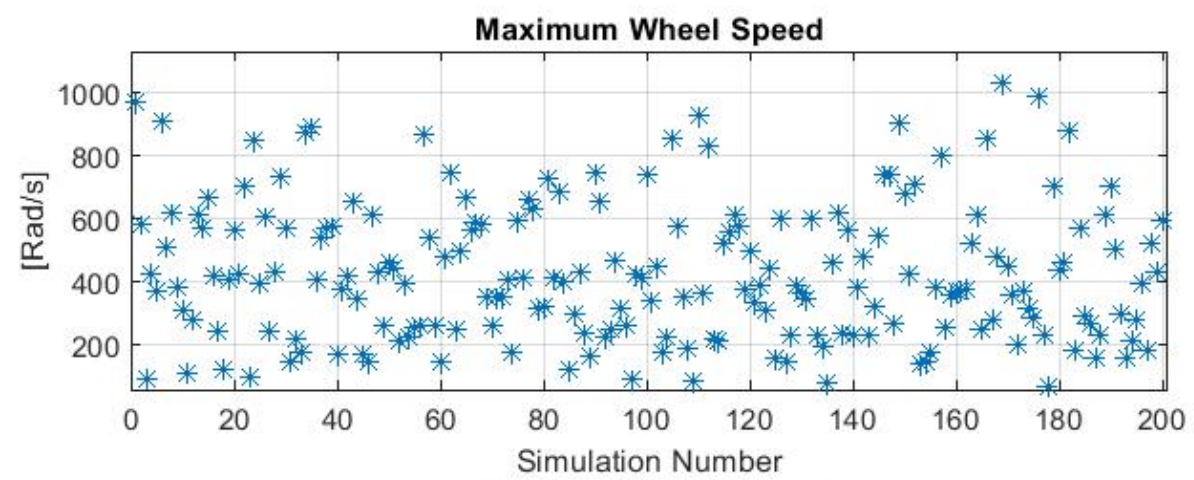

Minimum Wheel Speed

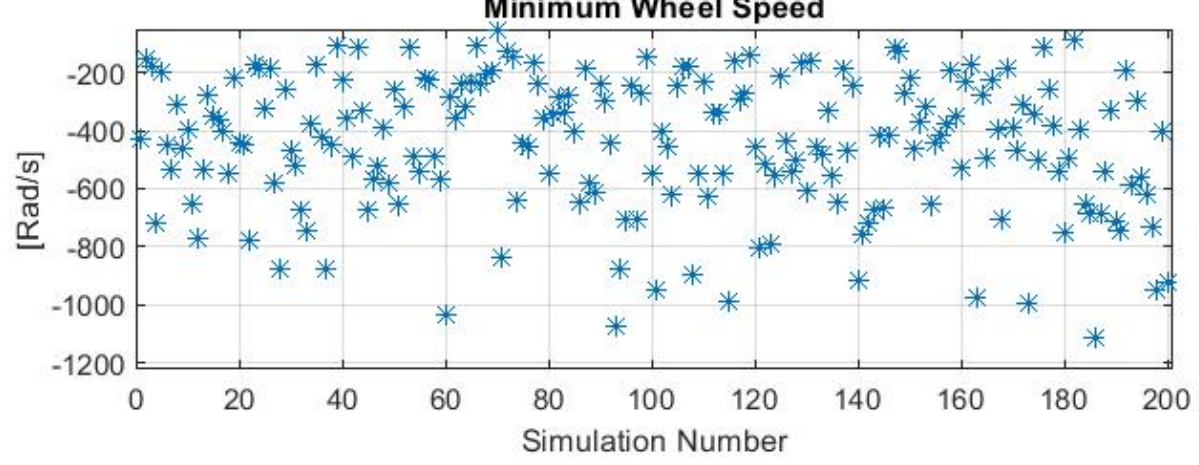

Figure 4.3.25: Maximum and Minimum Reaction Wheel Speed - Set 4 

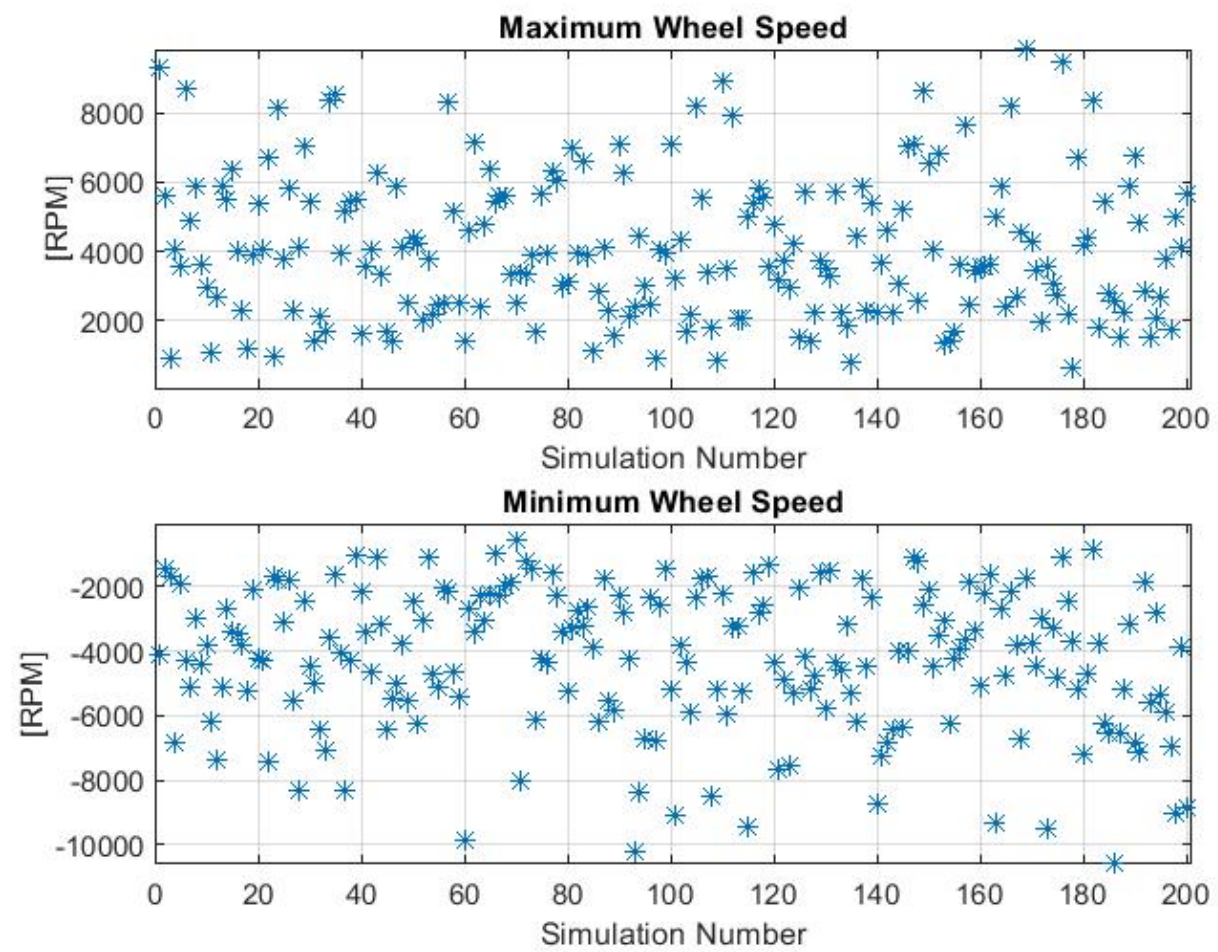

Figure 4.3.26: Maximum and Minimum Reaction Wheel Speed [RPM]- Set 4

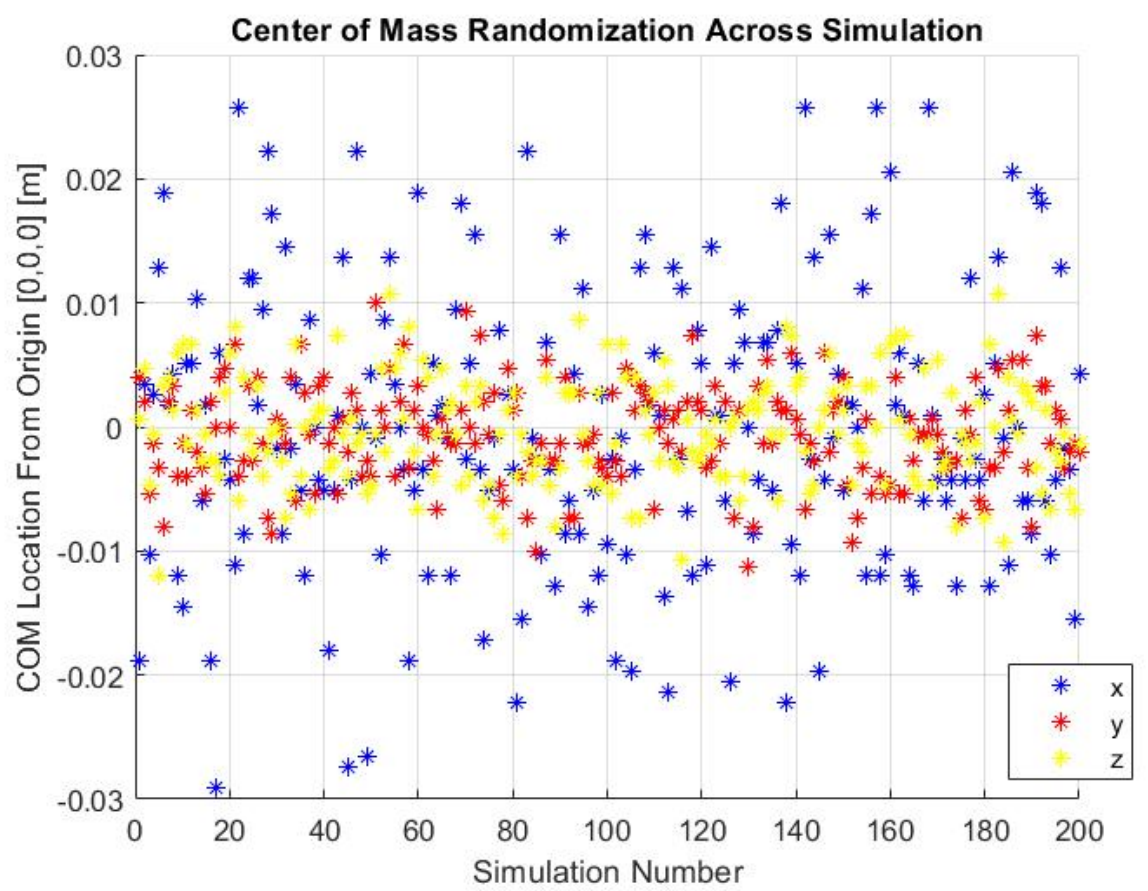

Figure 4.3.27: Center of Mass Distribution - Set 4 


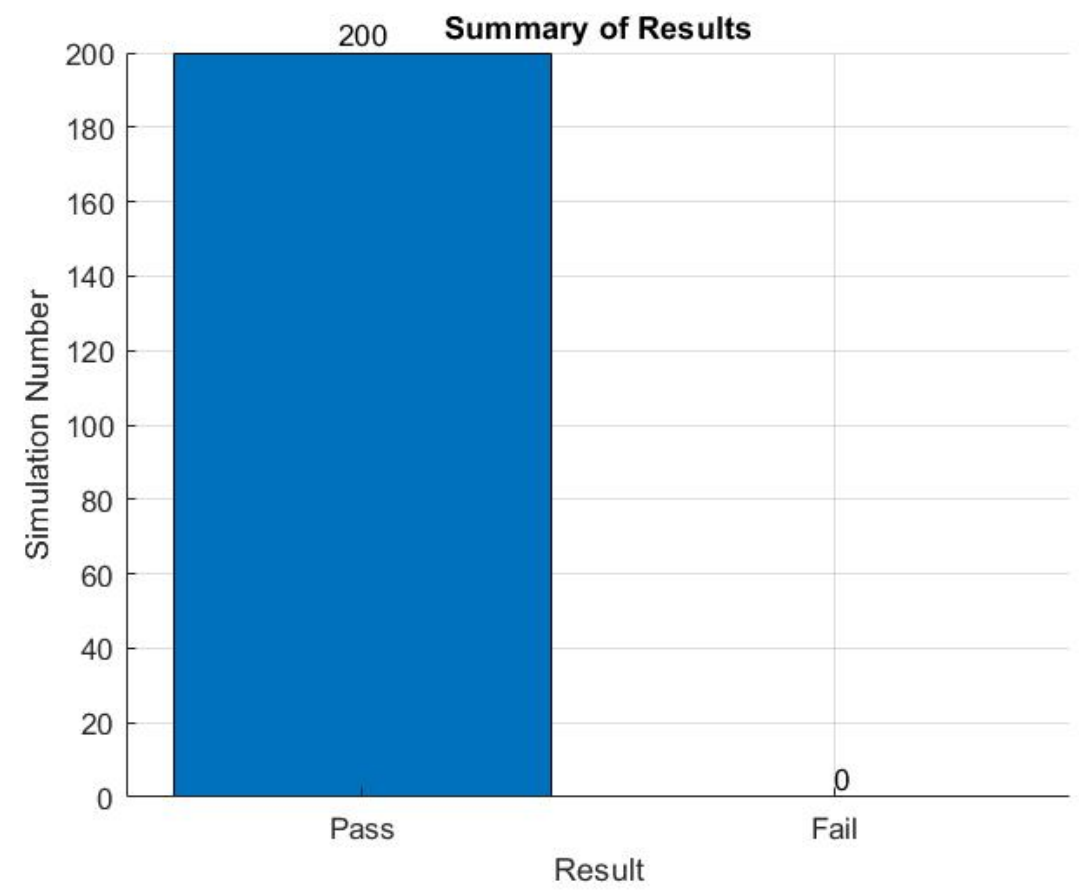

Figure 4.3.28: Summary of Results - Set 4 


\subsubsection{Simulation Set 5}

Table 4.6: Summary of Results for Simulation Set 5

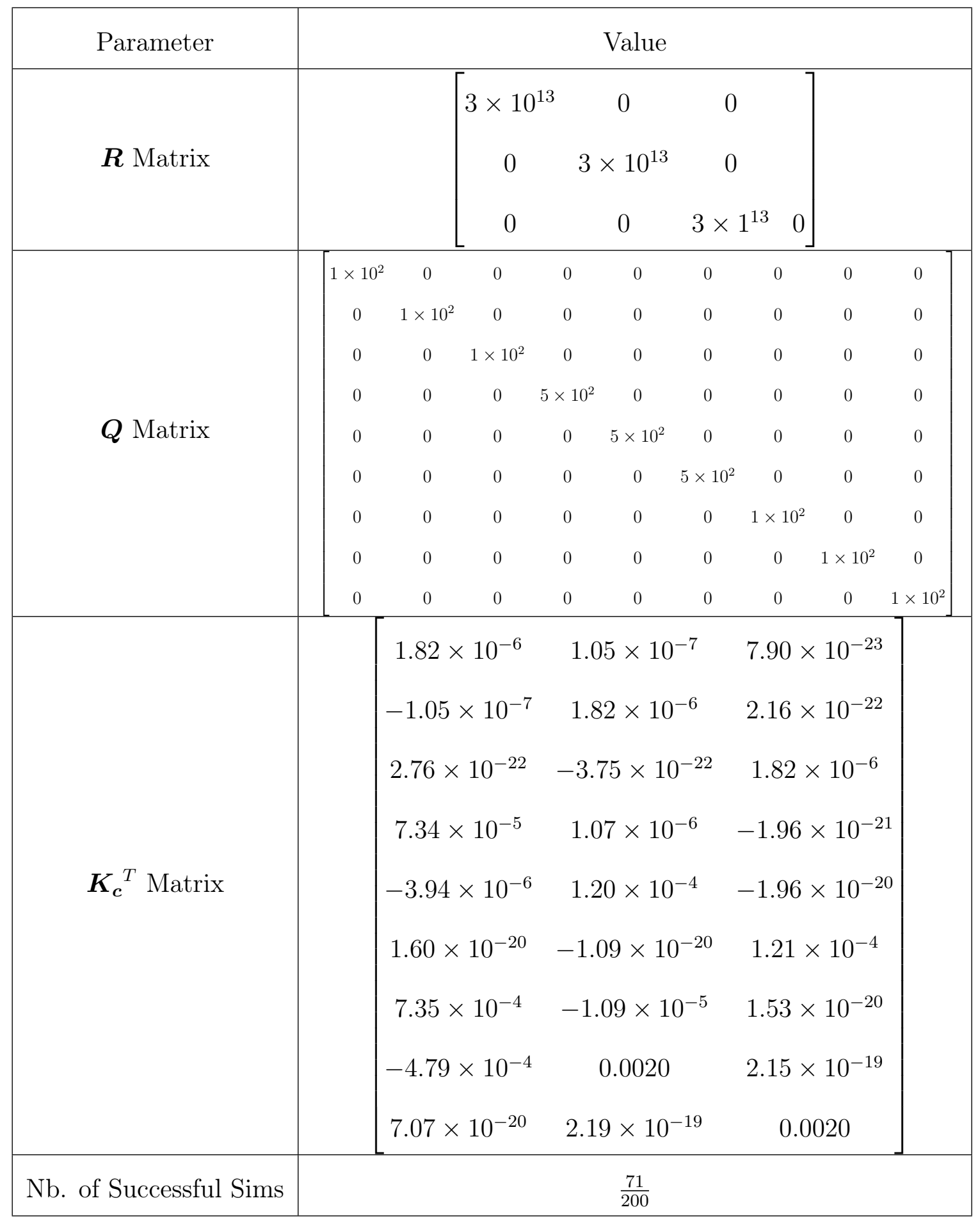




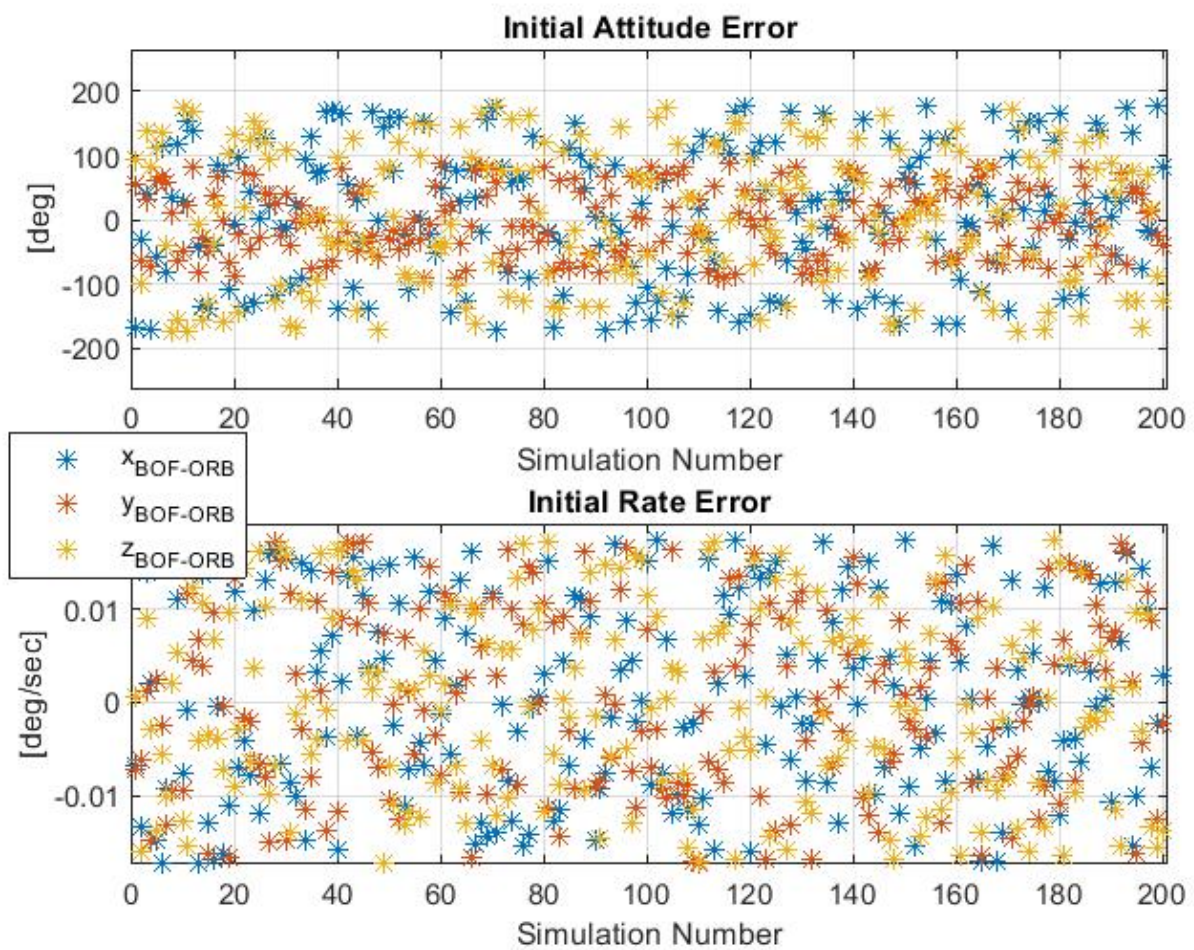

Figure 4.3.29: Initial Errors for Each Simulation - Set 5

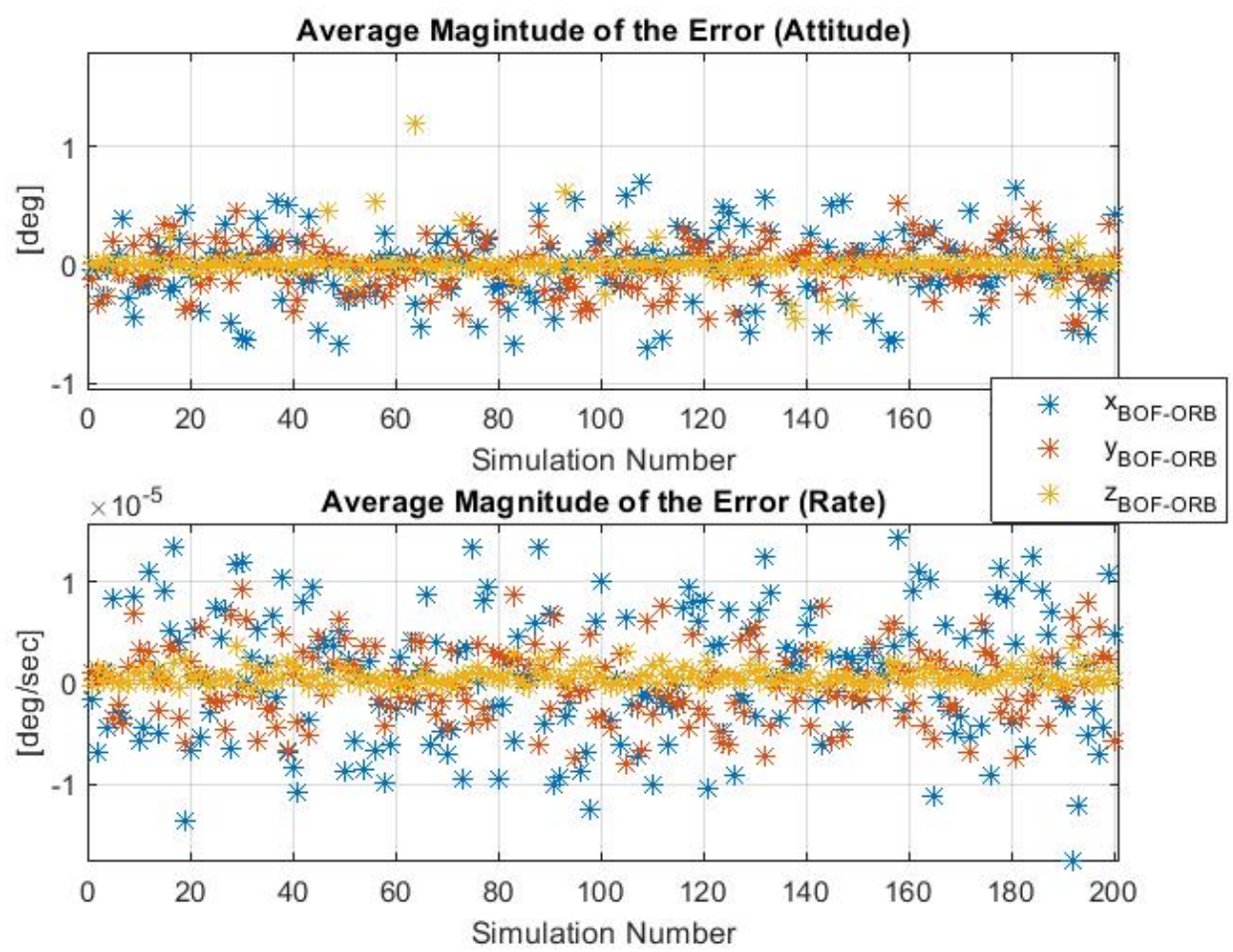

Figure 4.3.30: Average Errors for Each Simulation - Set 5 

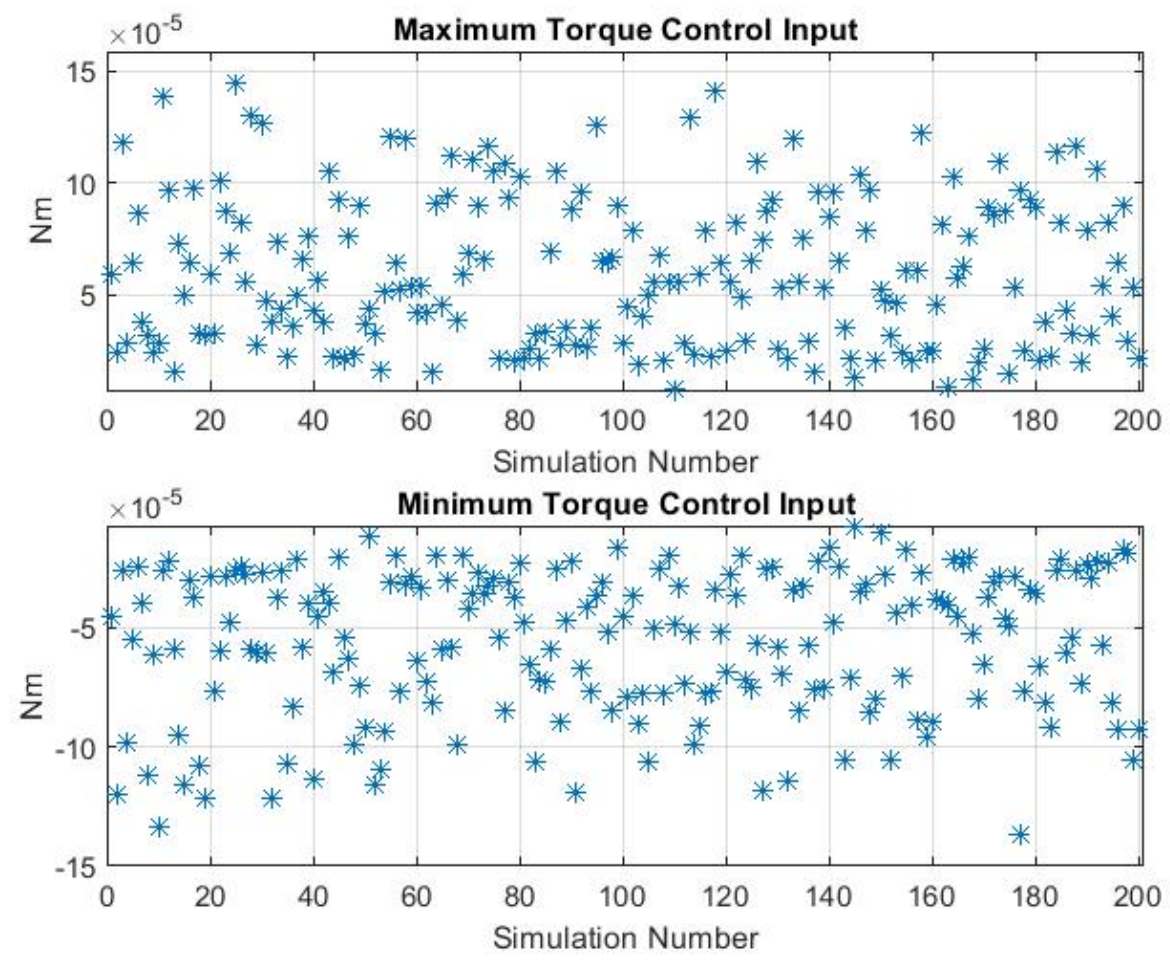

Figure 4.3.31: Maximum Control Inputs - Set 5
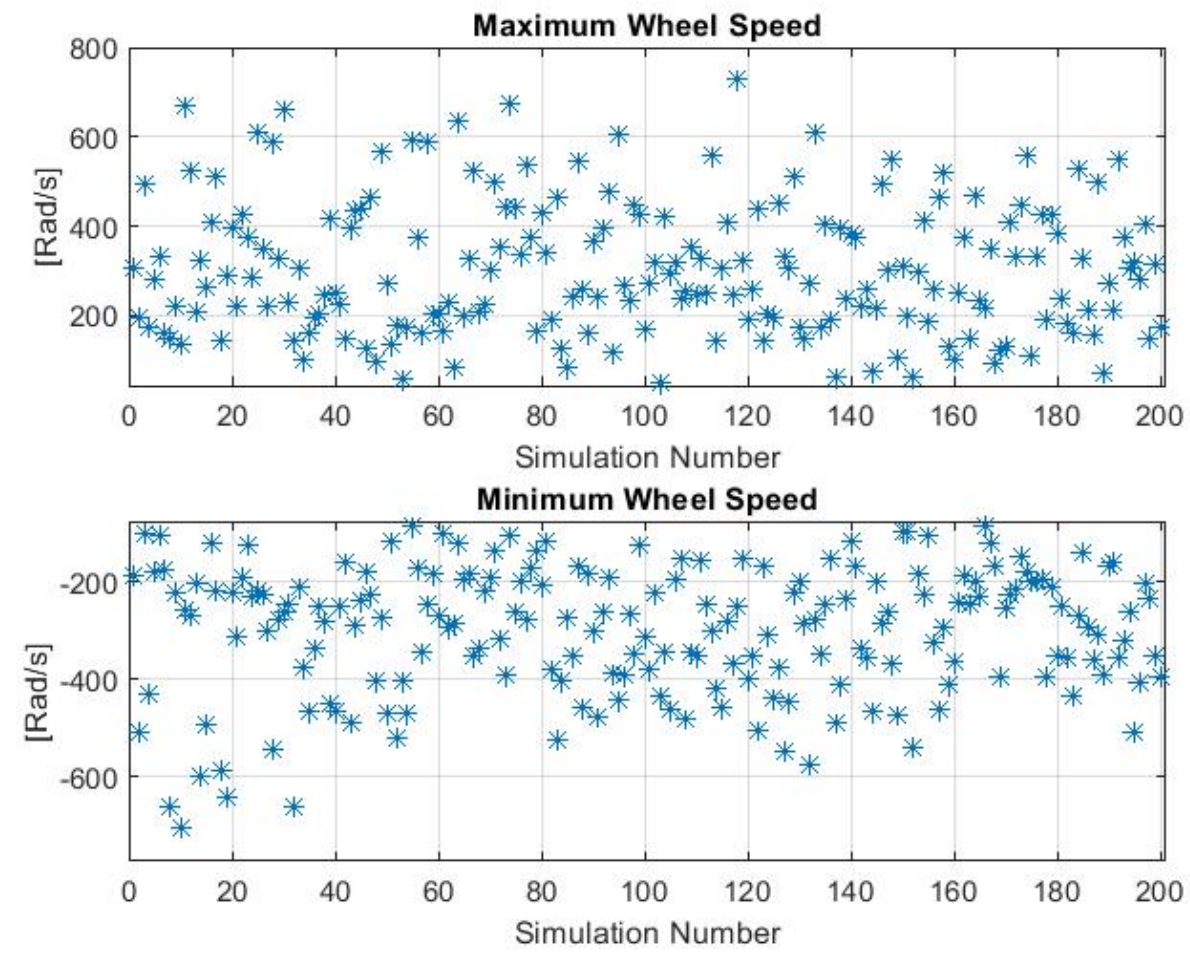

Figure 4.3.32: Maximum and Minimum Reaction Wheel Speed - Set 5 

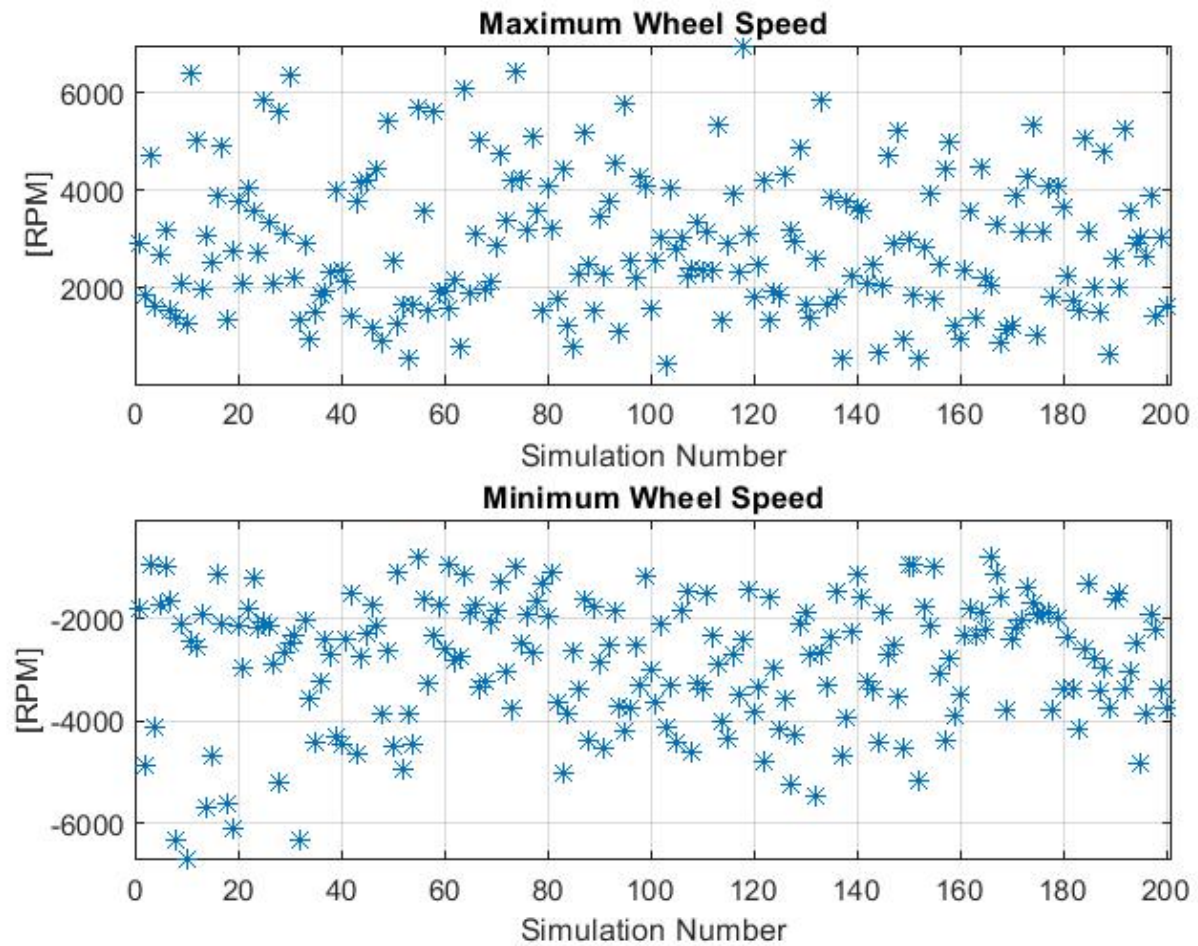

Figure 4.3.33: Maximum and Minimum Reaction Wheel Speed [RPM]- Set 5

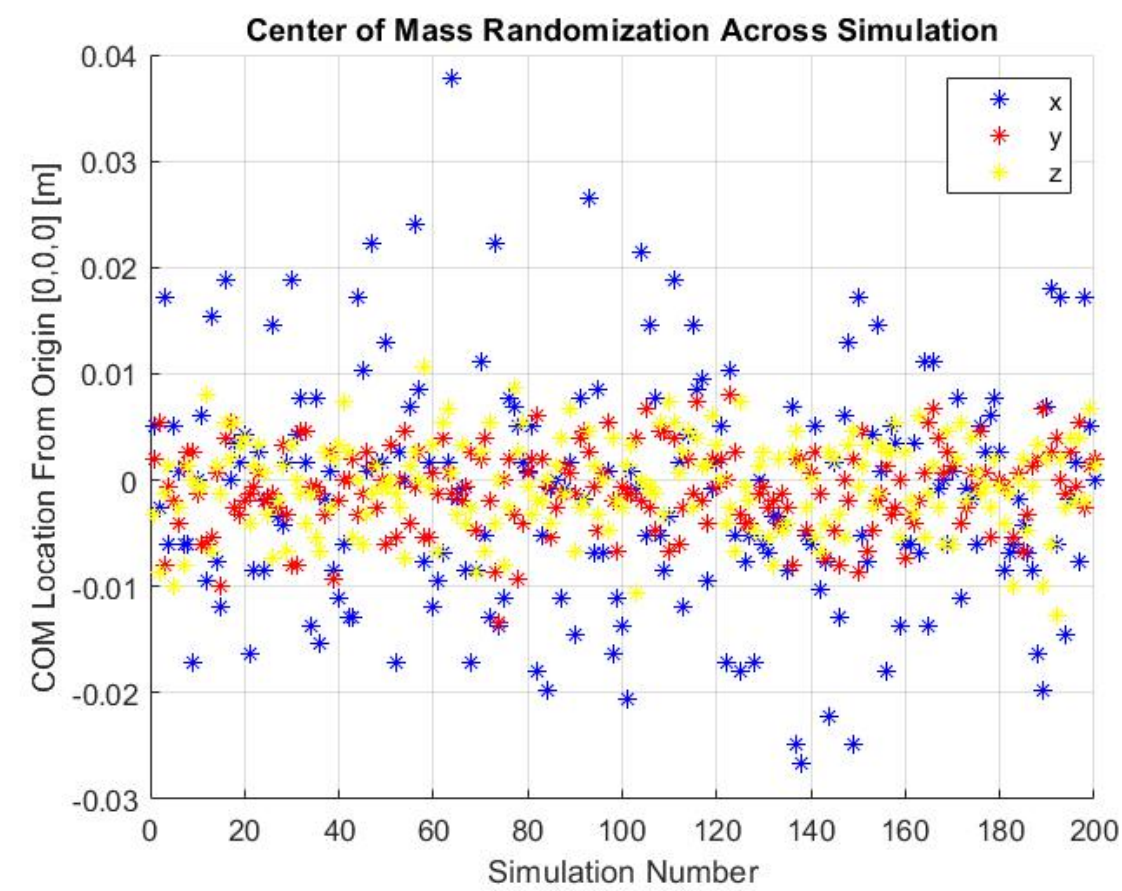

Figure 4.3.34: Center of Mass Distribution - Set 5 


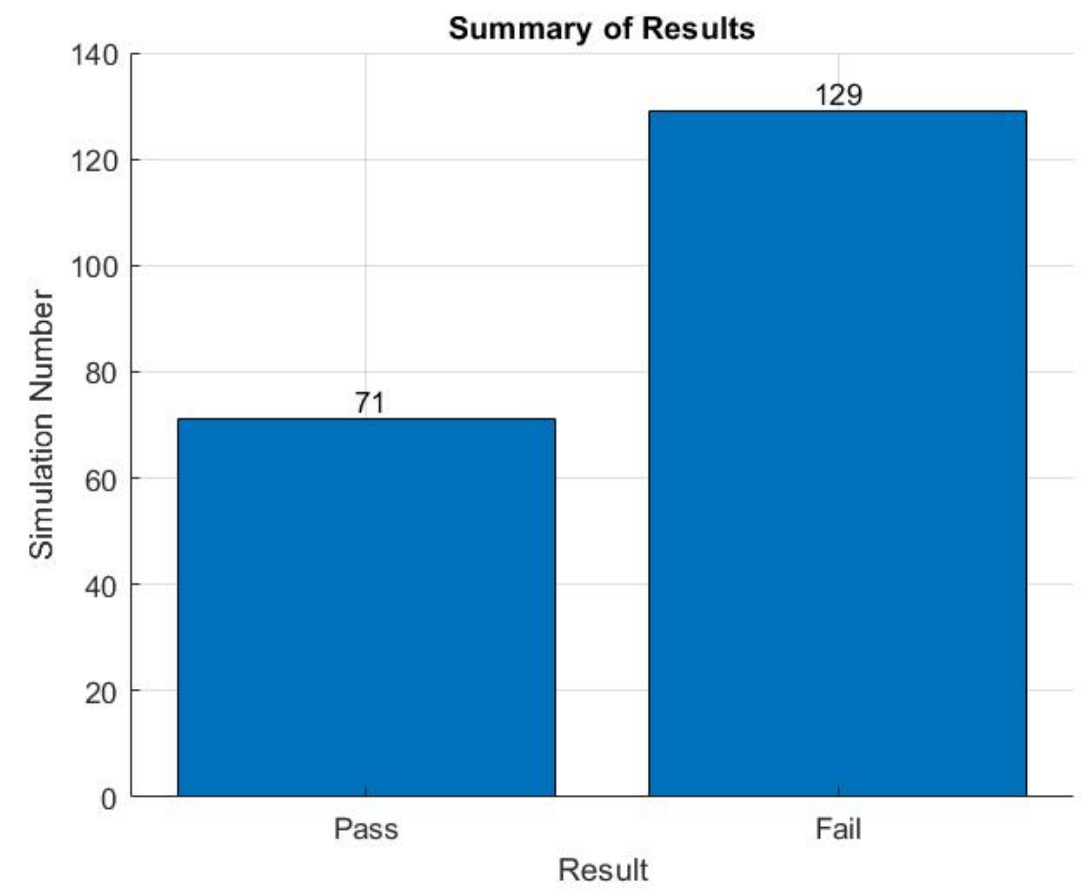

Figure 4.3.35: Summary of Results - Set 5

Clearly the fifth simulation set had a large number of failed simulations. To further investigate the failure, one of the failed simulations, simulation number 5 , was used to produce a more detailed summary of the RPY and angular rates throughout the simulation time. The following is a summary of the initial conditions for this simulation:

Table 4.7: Simulation 5 Initial Conditions

\begin{tabular}{|c|c|c|}
\hline InitialRPY $(\mathrm{deg})$ & InitialRates $(\mathrm{deg} / \mathrm{sec})$ & COMDistribution \\
\hline$\left[\begin{array}{c}-57.23 \\
62.76 \\
112.01\end{array}\right]$ & {$\left[\begin{array}{c}-0.0148 \\
0.0025 \\
-0.0138\end{array}\right]$} & {$\left[\begin{array}{c}0.0051 \\
-0.002 \\
-0.01\end{array}\right]$} \\
\hline
\end{tabular}



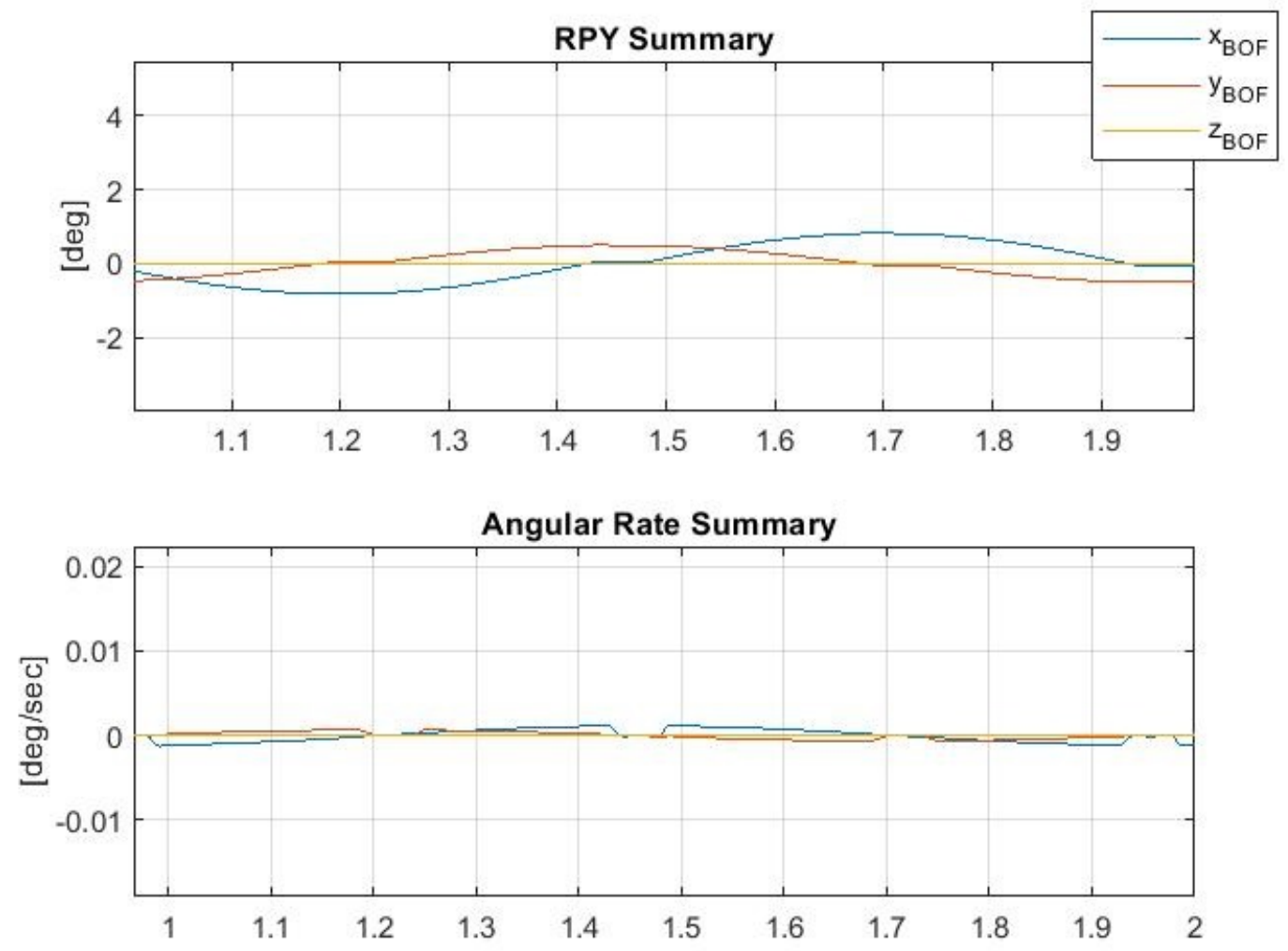

Figure 4.3.36: Attitude and Rates Throughout Orbit for Simulation 5 in Set 5

Since the section being analyzed is after the 1 orbit mark, figure (4.3.36) only shows that section of the orbit. As is seen in the figure, the attitude (RPY) of the satellite is still fluctuating and reaches peaks much larger $0.5 \mathrm{deg}$, thus classifying such simulation as a fail. This can also be seen in figure (4.3.30), where the average error for the attitude is larger than $\pm 0.5 \mathrm{deg}$ for many simulations in the set, thus deeming this controller to be unreliable. 


\section{Chapter 5}

\section{Conclusion}

The purpose of this project was to build and use a testing method to test different attitude control laws for the ESSENCE mission, which will be launched from the ISS. The theory and equations of motion were described and the existing model for the controller is shown. Controller block diagrams were displayed to further explain the controller logic. The simulation software is broken down into the different scripts used, and the results for the robust testing is shown. To validate the randomization of the test software, many plots were generated showing the initial attitude states, angular rates, and the center of mass distribution, for each simulation in each set. The maximum reaction wheel speeds were also plotted for each simulation to ensure over-saturation of the wheels does not occur. The results provide validation for 3 different controllers that have been proven to be robust and can stabilize the satellite through through a variety of different initial conditions, within the tight tolerances stated by the mission requirements.

This software setup is versatile and can be used and implemented to any other control law. Now that is has been validated with the LQR controller for the reaction wheel model, it can be implemented to the Bdot with bias controller. The only main differences would be changing the tolerances, simulation duration, and settling time, which are all variables in the test code, and so can be manipulated easily. Since the test software was broken down into separate scripts, when implementing it for the Bdot with bias controller, or any other control law, customising it has been made easy and straight forward. 


\section{Chapter 6}

\section{Bibliography}

[1] M. Alger, E. Gleeson, and W. Travis, ESSENCE ADCS Requirements Definition and Preliminary Design. Toronto: Ryerson University, University of Toronto Institute for Aerospace Studies (UTIAS), York University, 2 ed., 2018.

[2] N. R. Antares and M. Alger, ESSENCE Mission Description and Requirement Document. Toronto: Ryerson University, University of Toronto Institute for Aerospace Studies (UTIAS), York University, 2 ed., 2018.

[3] W. Travis, Flight Code Development and Monte-Carlo Testing of a Magnetically Actuated Attitude Control System. 350 Victoria St, Toronto, ON M5B 2K3: Ryerson Univerity, 1 ed., 2018.

[4] W. Travis, The Design and Analysis of an Attitude Determination Filter For CubeSat Mission. 350 Victoria St, Toronto, ON M5B 2K3: Ryerson Univerity, 1 ed., 2019.

[5] W. Travis, The Study of Utilizing a Linear Quadratic Regulator Controller Against a Pole Placement Controller for Satellite Attitude Control. 350 Victoria St, Toronto, ON M5B 2K3: Ryerson Univerity, 1 ed., 2019.

[6] D. Alexis, "Lqr control [online].available: http://www.kostasalexis.com/lqrcontrol.html.."

[7] W. Travis, "Analysis and design of attitude determination and control systems onboard micro satellites utilizing spaceborne synthetic aperture radar," 2021. 


\section{Appendix A}

\section{Linearization of A Matrix}

This appendix provides a step by step explanation of the linearization of the A matrix. To start off, the following two equations (A.1) and (A.2), are the ones being linearized.

$$
\begin{gathered}
\dot{\boldsymbol{x}}=\left[\begin{array}{c}
\dot{\boldsymbol{\eta}} \\
\dot{\boldsymbol{\epsilon}} \\
\dot{\boldsymbol{\omega}}
\end{array}\right]=\left[\begin{array}{c}
-\frac{1}{2} \boldsymbol{\epsilon}^{\boldsymbol{T}} \boldsymbol{\omega}_{B \boldsymbol{I}}^{B} \\
\frac{1}{2}\left(\boldsymbol{\epsilon}^{\times}+\eta \boldsymbol{I}_{\mathbf{3} \times \mathbf{3}}\right) \boldsymbol{\omega}_{B \boldsymbol{I}}^{B} \\
\boldsymbol{J}^{-\mathbf{1}}\left(-\boldsymbol{\omega}_{\boldsymbol{B} \boldsymbol{I}}^{B} \times \boldsymbol{J} \boldsymbol{\omega}_{\boldsymbol{B} \boldsymbol{I}}^{\boldsymbol{B}}+\boldsymbol{\tau}_{\boldsymbol{d i s}}\right)
\end{array}\right] \\
\boldsymbol{\tau}_{\boldsymbol{g} \boldsymbol{g}}=\frac{3 \mu}{\left\|\boldsymbol{r}_{B}(t)\right\|^{3}} \boldsymbol{r}_{B} \times \boldsymbol{J} \boldsymbol{r}_{\boldsymbol{B}}
\end{gathered}
$$

The first assumption to be made is the small angle approximation, and knowing that the quaternion error is double the small angle approximation. The attitude quaternion can be shown in equation (A.3). This equation shows us that the most important part from the attitude quaternion is the vector component, and so the scalar component can be omitted in the linearization.

$$
\delta q=\left[\begin{array}{c}
1 \\
\Delta \epsilon
\end{array}\right]
$$

The main objective of the control laws stated in this report is to align the attitude of the body frame with the orbital frame with minimal deviation, and the equilibrium and reference point used for the linearization is stated below in equation (A.4) 


$$
\begin{gathered}
\dot{\boldsymbol{x}}=f\left(x=x_{e}\right)=0 \\
\dot{\boldsymbol{x}}=\left[\begin{array}{c}
\dot{\epsilon_{x}} \\
\dot{\epsilon_{y}} \\
\dot{\epsilon_{z}} \\
\dot{\omega}_{x} \\
\dot{\omega}_{y} \\
\dot{\omega}_{z}
\end{array}\right]=0
\end{gathered}
$$

Next, the cross coupled term $-\boldsymbol{\omega}_{\boldsymbol{B} \boldsymbol{I}}^{B} \times \boldsymbol{J} \boldsymbol{\omega}_{\boldsymbol{B I}}^{\boldsymbol{B}}$ is used as the initial point of the linearization.

First, the shape of the spacecraft is assumed to be symmetrical, and so the moment of inertia matrix of the spacecraft can be defined in equation (A.5)

$$
\boldsymbol{J}=\left[\begin{array}{ccc}
I_{x x} & 0 & 0 \\
0 & I_{y y} & 0 \\
0 & 0 & I_{z z}
\end{array}\right]
$$

The cross coupled term can then be linearized in the following set of equations:

$$
\begin{gathered}
-\boldsymbol{\omega}^{\times} \boldsymbol{J} \boldsymbol{\omega}=-\left[\begin{array}{l}
\omega_{x} \\
\omega_{y} \\
\omega_{z}
\end{array}\right] \times\left[\begin{array}{l}
\omega_{x} I_{x x} \\
\omega_{y} I_{y y} \\
\omega_{z} I_{z z}
\end{array}\right]=\left[\begin{array}{l}
\left(I_{y y}-I_{z z}\right) \omega_{y} \omega_{z} \\
\left(I_{z z}-I_{x x}\right) \omega_{x} \omega_{z} \\
\left(I_{x x}-I_{y y}\right) \omega_{x} \omega_{y}
\end{array}\right] \\
\dot{\boldsymbol{\omega}}_{c \boldsymbol{c}}=\boldsymbol{J}^{-\mathbf{1}}\left(-\boldsymbol{\omega}^{\times} \boldsymbol{J} \boldsymbol{\omega}\right)=\left[\begin{array}{c}
\frac{I_{y y}-I_{z z}}{I_{x x}} \omega_{y} \omega_{z} \\
\frac{I_{z z}-I_{x x}}{I_{y y}} \omega_{x} \omega_{z} \\
\frac{I_{x x}-I_{y y}}{I_{z z}} \omega_{x} \omega_{y}
\end{array}\right] \\
\delta \dot{\boldsymbol{\omega}}_{c \boldsymbol{c}}=\left[\begin{array}{c}
\frac{I_{y y}-I_{z z}}{I_{x x}}\left(\delta \omega_{y} \omega_{z, e}+\delta \omega_{z} \omega_{y, e}\right) \\
\frac{I_{z z}-I_{x x}}{I_{y y}}\left(\delta \omega_{x} \omega_{z, e}+\delta \omega_{z} \omega_{x, e}\right) \\
\frac{I_{x x}-I_{y y}}{I_{z z}}\left(\delta \omega_{x} \omega_{x, e}+\delta \omega_{y} \omega_{y, e}\right)
\end{array}\right]
\end{gathered}
$$

In order to simplify the notation of the equations, set the following,

$$
\omega_{0}=\sqrt{\frac{\mu}{r^{3}}}
$$




$$
\sigma=\left[\begin{array}{l}
\sigma_{x} \\
\sigma_{y} \\
\sigma_{z}
\end{array}\right]=\left[\begin{array}{c}
\frac{I_{y y}-I_{z z}}{I_{x x}} \\
\frac{I_{z z}-I_{x x}}{I_{y y}} \\
\frac{I_{x x}-I_{y y}}{I_{z z}}
\end{array}\right]
$$

By substituting the simplifications in equations (A.9) and (A.10), along with the equilibrium conditions for the $\omega_{x, e}, \omega_{y, e}, \omega_{z, e}$, which are all equal to zero, except for the $\omega_{z, e}$ term, which is eqaul to the orbital velocity $\omega_{0}$, the linearized cross-coupled term can be determined in equation (A.11).

$$
\delta \dot{\boldsymbol{\omega}}_{\boldsymbol{c c}}=\left[\begin{array}{c}
\sigma_{x} \delta \omega_{y} \omega_{0} \\
\sigma_{y} \delta \omega_{x} \omega_{0} \\
0
\end{array}\right]
$$

Once the cross-coupled term is linearized and presented above in equation (A.11), the gravity gradient torque presented in equation (A.2) can now be linearized. The assumptions made in this step is that the spacecraft maintains a circular orbit around the Earth, and the orbital vector $\hat{\boldsymbol{r}}$, is considered to be the unit vector of the $\mathrm{x}$-axis in the orbital frame. The method used for linearizing this gradient is to express the torque generated by the gravity gradient as an angular acceleration, and to rearrange equation (A.9) and express it in terms of $\mu$. The linearization process is presented in the following set of equations.

$$
\begin{aligned}
& \boldsymbol{\tau}_{\boldsymbol{g} \boldsymbol{g}}=\boldsymbol{J} \delta \dot{\boldsymbol{\omega}}_{\boldsymbol{g g}}=\frac{3 \mu}{\|\boldsymbol{r}(t)\|^{3}} \delta \hat{\boldsymbol{r}}^{\times} \boldsymbol{J} \delta \hat{\boldsymbol{r}} \\
& \delta \dot{\boldsymbol{\omega}}_{\boldsymbol{g g}}=3 \omega_{0}^{2} \boldsymbol{J}^{-\mathbf{1}}\left(\delta \hat{\boldsymbol{r}}^{\times} \boldsymbol{J} \delta \hat{\boldsymbol{r}}\right) \\
& \delta \dot{\boldsymbol{\omega}}_{\boldsymbol{g} \boldsymbol{g}}=3 \omega_{0}^{2}\left[\begin{array}{ccc}
\frac{1}{I_{x x}} & 0 & 0 \\
0 & \frac{1}{I_{y y}} & 0 \\
0 & 0 & \frac{1}{I_{z z}}
\end{array}\right]\left(\left[\begin{array}{c}
1 \\
-2 \delta \epsilon_{z} \\
2 \delta \epsilon_{y}
\end{array}\right] \times\left[\begin{array}{ccc}
I_{x x} & 0 & 0 \\
0 & I_{y y} & 0 \\
0 & 0 & I_{z z}
\end{array}\right]\left[\begin{array}{c}
1 \\
-2 \delta \epsilon_{z} \\
2 \delta \epsilon_{y}
\end{array}\right]\right) \\
& \delta \dot{\boldsymbol{\omega}}_{\boldsymbol{g g}}=3 \omega_{0}^{2}\left[\begin{array}{ccc}
\frac{1}{I_{x x}} & 0 & 0 \\
0 & \frac{1}{I_{y y}} & 0 \\
0 & 0 & \frac{1}{I_{z z}}
\end{array}\right]\left[\begin{array}{c}
-4 \delta \epsilon_{z} \delta \epsilon_{y} I_{z z}+4 \delta \epsilon_{y} \delta \epsilon_{z} I_{y y} \\
2 \delta \epsilon_{y} I_{z z}+2 \delta \epsilon_{y} I_{x x} \\
-2 \delta \epsilon_{z} I_{y y}+2 \delta \epsilon_{z} I_{x x}
\end{array}\right] \\
& \delta \dot{\boldsymbol{\omega}}_{\boldsymbol{g g}}=3 \omega_{0}^{2}\left[\begin{array}{c}
0 \\
2 \delta \epsilon_{y} \frac{I_{x x}-I_{z z}}{I_{y y}} \\
2 \delta \epsilon_{z} \frac{I_{x x}-I_{y y}}{I_{z z}}
\end{array}\right]
\end{aligned}
$$


Substituting in the equation (A.10), and ignoring the higher order terms, the final linearized equation for the gravity gradient torque can be expressed in equation (A.17)

$$
\delta \dot{\boldsymbol{\omega}}_{\boldsymbol{g g}}=6 \omega_{0}^{2}\left[\begin{array}{c}
0 \\
-\sigma_{y} \delta \epsilon_{y} \\
\sigma_{z} \delta \epsilon_{z}
\end{array}\right]
$$

Finally, the kinematics are linearized, and the linearization process is shown throughout the following set of equations:

$$
\begin{gathered}
\dot{\boldsymbol{\epsilon}}=\frac{1}{2}\left(\boldsymbol{\epsilon}^{\times}+\eta \boldsymbol{I}_{\mathbf{3} \times \mathbf{3}}\right) \boldsymbol{\omega}_{\boldsymbol{B} \boldsymbol{I}}^{\boldsymbol{B}} \\
\dot{\boldsymbol{\epsilon}}=\frac{1}{2}\left[\begin{array}{ccc}
\eta & -\epsilon_{z} & \epsilon_{y} \\
\epsilon_{z} & \eta & -\epsilon_{x} \\
-\epsilon_{y} & \epsilon_{x} & \eta
\end{array}\right]\left[\begin{array}{l}
\omega_{x} \\
\omega_{y} \\
\omega_{z}
\end{array}\right] \\
\dot{\boldsymbol{\epsilon}}=\frac{1}{2}\left[\begin{array}{c}
\eta \omega_{x}-\epsilon_{z} \omega_{y}+\epsilon_{y} \omega_{z} \\
\epsilon_{z} \omega_{x}+\eta \omega_{y}-\epsilon_{x} \omega_{z} \\
-\epsilon_{y} \omega_{x}+\epsilon_{x} \omega_{y}+\eta \omega_{z}
\end{array}\right] \\
\dot{\boldsymbol{\epsilon}}=\frac{1}{2}\left[\begin{array}{c}
\delta \eta \omega_{x, e}+\eta \delta \omega_{x}-\delta \epsilon_{z} \omega_{y, e}-\delta \omega_{y} \epsilon_{z, e}+\delta \epsilon_{y} \omega_{z, e}+\delta \omega_{z} \epsilon_{y, e} \\
\delta \epsilon_{z} \omega_{x, e}+\delta \omega_{x} \epsilon_{z, e}+\delta \eta \omega_{y, e}+\eta \delta \omega_{y}-\delta \epsilon_{x} \omega_{z, e}-\epsilon_{x, e} \delta \omega_{z, e} \\
-\delta \epsilon_{y} \omega_{x, e}-\delta \omega_{x} \epsilon_{y, e}+\delta \epsilon_{x} \omega_{y, e}+\epsilon_{x, e} \delta \omega_{y}+\delta \eta \omega_{z, e}+\eta \delta \omega_{z}
\end{array}\right]
\end{gathered}
$$

Then introducing the equilibrium state, the linearized kinematics can be expressed in equation (A.22)

$$
\dot{\boldsymbol{\epsilon}}=\frac{1}{2}\left[\begin{array}{c}
\delta \omega_{x}+\omega_{0} \delta \epsilon_{y} \\
\delta \omega_{y}-\omega_{0} \delta \epsilon_{x} \\
\delta \omega_{z}
\end{array}\right]
$$

Now combining equations (A.11), (A.17), and (A.22), the final linearized A matrix is expressed in equation (A.23)

$$
\boldsymbol{A}=\left[\begin{array}{cccccc}
0 & \omega_{0} & 0 & \frac{1}{2} & 0 & 0 \\
-\omega_{0} & 0 & 0 & 0 & \frac{1}{2} & 0 \\
0 & 0 & 0 & 0 & 0 & \frac{1}{2} \\
0 & 0 & 0 & 0 & \sigma_{x} \omega_{0} & 0 \\
0 & -6 \omega_{0}^{2} \sigma_{y} & 0 & \sigma_{y} \omega_{0} & 0 & 0 \\
0 & 0 & 6 \omega_{0}^{2} \sigma_{z} & 0 & 0 & 0
\end{array}\right]
$$

